\title{
12. PALYNOLOGICAL STRATIGRAPHY OF DEEP SEA DRILLING PROJECT SITE 416
}

\author{
G. L. Williams and J. P. Bujak, Atlantic Geoscience Centre, Bedford Institute of Oceanography, \\ Dartmouth, N.S., Canada
}

\begin{abstract}
Samples from DSDP Hole $416 \mathrm{~A}$, Cores 53 to 9 , contain both spores and dinoflagellate cysts. The palynomorphs indicate the presence of Kimmeridgian-Portlandian sediments (Cores 52 and 51) which are overlain by Berriasian (Cores 50 to 32), Valanginian (Cores 32 to 10 ), and Hauterivian (Core 9) strata.
\end{abstract}

\section{INTRODUCTION}

Ninety samples from DSDP Cores 416A-53 to 416A-9 (Figure 1) were palynologically analyzed. The oldest sediments dated using spores and dinoflagellates were Kimmeridgian-Portlandian. These are overlain by Berriasian, Valanginian, and Hauterivian sediments. Samples below Core 416A-53 and Core 416A-9 were received too late to include data from them in this report.

Appendix A alphabetically lists all palynomorph species recorded from Hole 416A and references the specimens illustrated in this paper. All illustrated specimens are curated at the Geological Survey of Canada, Dartmouth, Nova Scotia, under GSC type Numbers 56697 to 56793 .

Details of species occurrences and age assignments are given in the following biostratigraphic section.

\section{BIOSTRATIGRAPHY}

\section{Kimmeridgian-Portlandian}

The interval between Samples 416A-52-3, $99 \mathrm{~cm}$ and $416 \mathrm{~A}-51-1,17 \mathrm{~cm}$ is dated palynologically as Upper Jurassic (undifferentiated Kimmeridgian-Portlandian.)

The specimens of Senoniasphaera jurassica differ from the type material in possessing a perforate periphragm. They are identical to specimens common in the Oxfordian-Kimmeridgian of the Scotian Shelf, offshore south-eastern Canada (Bujak and Williams, 1977) and the Upper Jurassic of the COST B-2 well, drilled in the Baltimore Canyon, offshore eastern United States (personal observation). Some specimens of $S$. jurassica described from the Kimmeridgian of southern England by Ioannides et al. (1977) also possess small perforations in the periphragm. The species Ctenidodinium panneum is known only from the Portlandian of southern England (Norris, 1965) and the Kimmeridgian-Portlandian of the Scotian Shelf (Bujak and Williams, 1977). Systematophora sp. B of Ioannides et al. (1977) from the Kimmeridgian of southern England may be conspecific with specimens described as Systematophora turonica from the Callovian-Kimmeridgian of the Scotian Shelf by Bujak and Williams (1977). ?Prolixosphaeridium torynum has a known stratigraphic range of Kimmeridgian to

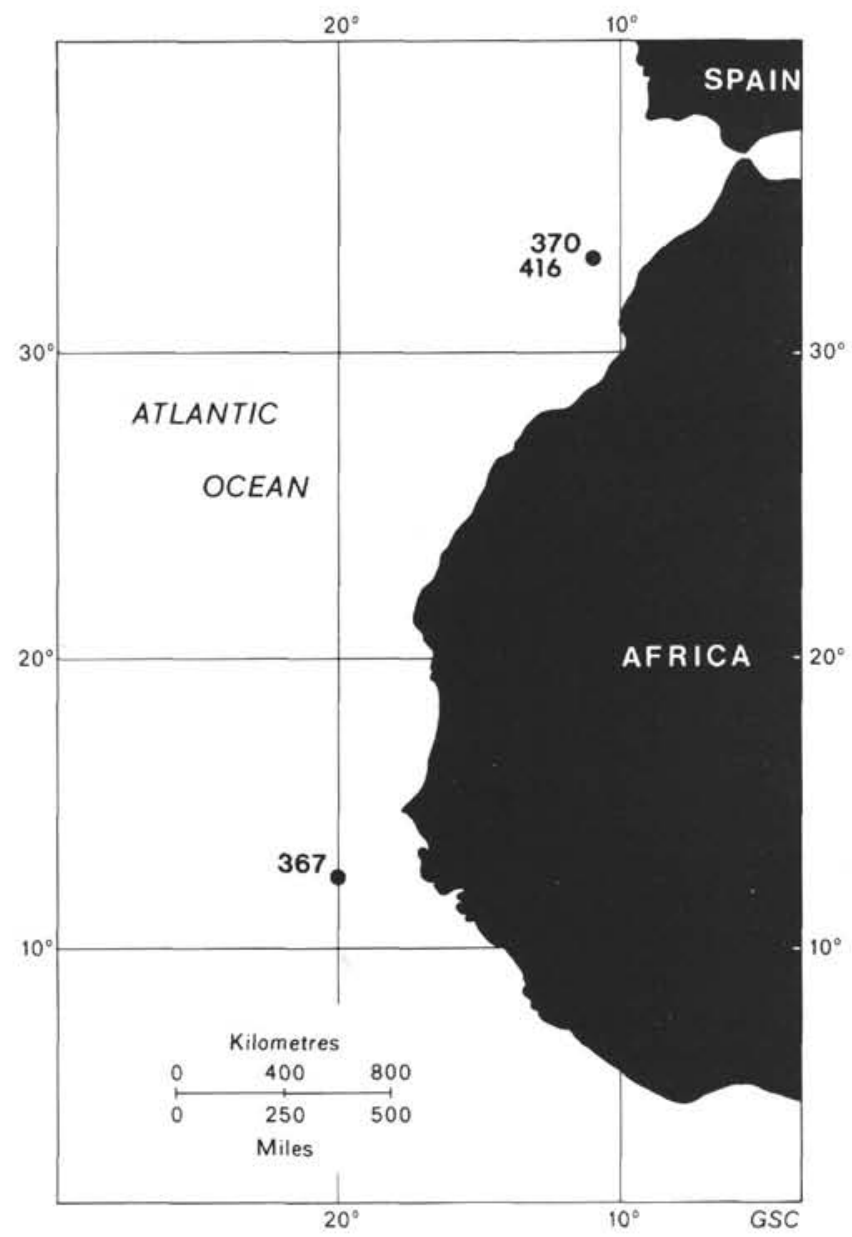

Figure 1. Location map, DSDP Sites 367, 370, and 416.

Neocomian (Cookson and Eisenack, 1960b; Ioannides et al., 1977). The stratigraphic range of Muderongia simplex, which was first described from the Valanginian-Barremian by Alberti (1961), has now been extended into the uppermost Oxfordian (Bujak and Williams, 1977). Hystrichodinium sp. A of Ioannides et al. (1977) from the Kimmeridgian of southern England is conspecific with specimens incorrectly named Hystrichodinium 
pulchrum from the Kimmeridgian-Portlandian of the Scotian Shelf-Grand Banks by Bujak and Williams (1977).

Details of species occurrences are listed in Table 1.

\section{Berriasian}

Berriasian sediments extend from Samples 416A$50-1,49 \mathrm{~cm}$ to $416 \mathrm{~A}-32-5,11 \mathrm{~cm}$, based on palynological analysis. Sample richness for spores and dinoflagellates varies greatly within this interval. Approximately 30 dinoflagellate species and over 20 spore species were recorded. Spores include Appendicisporites jansonii, Cicatricosisporites augustus, Cicatricosisporites hughesi, Cicatricosisporites auritus, Ephedripites sp. C, Leptolepidites psarosus, Pilosisporites trichopapillosus, Trilobosporites apiverrucatus, and Trilobosporites jurassicus. Except for the presence of $T$. jurassicus, this assemblage is typical of those present in the Neocomian of offshore eastern Canada (Bujak and Williams, in press).

The dinoflagellate assemblages are usually diverse for sediments of this age that have been previously described. Species present can be placed in one of three groups. First are species that have not been described from sediments older than Lower Cretaceous. These include Endoscrinium campanulum (Berriasian-Coniacian), Hystrichosphaeridium recurvatum (ValanginianMaestrichtian), Kleithriasphaeridium eoinodes (Berriasian-Aptian), Polysphaeridium warrenii (BerriasianAptian), Tanyosphaeridium boletum (only described to date from the Barremian), and Trichodinium castaneum (Valanginian-Campanian). Second are species that are known from the Jurassic and Cretaceous. These include the dinoflagellates Cyclonephelium distinctum, Muderongia simplex, Muderongia tetracantha, Gonyaulacysta fastigiata, and Tenua hystrix, and the spores Aequitriradites verrucosus, Callialasporites dampieri, Callialasporites trilobatus, Cicatricosisporites australiensis, Corollina torosus, Klukisporites foveolatus, Klukisporites pseudoreticulatus, Leptolepidites psarosus, Pilosisporites trichopapillosus, and Trilobosporites apiverrucatus. Third, rare species occur that have previously been described only from the Jurassic, and that may range into the Lower Cretaceous or may represent reworking. These include Ctenidodinium culmulum (Kimmeridgian-Portlandian), Gonyaulacysta ambigua (Callovian-Portlandian), Gonyaulacysta granulata (Oxfordian-Portlandian), Systematophora fasciculigera (Oxfordian-Kimmeridgian), and Systematophora orbifera (Callovian-Kimmeridgian). Scriniocassis dictyotus, which also occurs in this interval, was first described from the Oxfordian-Tithonian of Australia and New Guinea by Cookson and Eisenack (1960) and was subsequently recorded by Habib (1972) from the "Early Cretaceous" and "probably Valanginian" of Site 105, western North Atlantic.

Details of species occurrences are listed in Table 2.

\section{Valanginian}

Valanginian sediments extend from Sample 416A$32-3,129 \mathrm{~cm}$ to $416 \mathrm{~A}-10-1,83 \mathrm{~cm}$, on the basis of paly- nological analysis. Most samples contain both spores and dinoflagellates; some are devoid of dinoflagellates, whereas a few samples contain extremely rich, diverse spore and dinoflagellate assemblages (e.g., 416A-12-4, $112-116 \mathrm{~cm}$; 416A-31-3, 70-74 cm; 416A-28-3, 58-62 $\mathrm{cm})$.

With the exception of rare specimens of Ctenidodinium culmulum, Ctenidodinium schizoblatum, Gonyaulacysta ambigua, and Gonyaulacysta granulata, which have previously been described only from the Jurassic, the dinoflagellate assemblages are diagnostic of Lower Cretaceous strata. Typical Cretaceous species not known from pre-Valanginian strata include Ctenidodinium elegantulum, Hystrichosphaeridium recurvatum, Oligosphaeridium complex, ?Spiniferites dentatus, and Systematophora complicata.

Other species which occur in this interval with stratigraphic ranges restricted to the Cretaceous include Achomosphaera neptuni (Berriasian-Aptian), Cribroperidinium orthoceras (Berriasian-Cenomanian), Cribroperidinium sepimentum (?Hauterivian-Aptian), Diacanthum hollisteri (Berriasian-Valanginian), Endoscrinium campanulum (Berriasian-Coniacian), Kleithriasphaeridium eoinodes (Berriasian-Aptian), Phoberocysta neocomica (Berriasian-Aptian), Pseudoceratium pelliferum (Berriasian-Barremian), Systematophora schindewolfii (Berriasian-Aptian), Tenua anaphrissa (?Barremian-Aptian), and Wallodinium krutzschi (Berriasian-Barremian). Details of species occurrences are given in Table 3.

\section{Hauterivian}

Sample 416A-9-4, 31-35 cm is dated Hauterivian from comparison with the dinoflagellate assemblages present at Site 370 (Williams, in press). Species having earliest occurrences include Cyclonephelium tabulatum, described by Williams as Cyclonephelium attadalicum, Meiourogonyaulax stoveri, and Oligosphaeridium pulcherrimum. Details of species occurrences are in Table 4.

\section{ACKNOWLEDGMENTS}

The authors are grateful to C. A. Noakes and D. A. Umpleby for their constructive criticism of the paper.

\section{REFERENCES}

Alberti, G., 1961. Zur Kenntnis Mesozoischer und Alttertiärer Dinoflagellaten und Hystrichosphaerideen von Nord- und Mitteldeutchland sowie einigen anderen Europäischen Gebieten, Palaeontographica, v. 116, p. 1-58.

Bujak, J. P. and Williams, G. L., 1977. Jurassic palynostratigraphy of off-shore eastern Canada. In Swain, F. M. (Ed.), Stratigraphic micropaleontology of Atlantic Basin and Borderlands: Amsterdam (Elsevier Sci. Publ. Co.), p. 321-339.

Bujak, J. P. and Williams, G. L., in press. Cretaceous palynostratigraphy of offshore southeastern Canada, Geological Survey of Canada Bulletin.

Cookson, I. C. and Eisenack, A., 1960. Upper Mesozoic microplankton from Australia and New Guinea, Palaeontology, v. 2, p. 243-261.

Habib, D., 1972. Dinoflagellate stratigraphy, Leg 11, Deep Sea Drilling Project. In Hollister, C. D., Ewing, J. I., et 
TABLE 1

Details of Spore and Dinoflagellate Species Occurrences in the Kimmeridgian-Portlandian Sequence, Site 416

\begin{tabular}{|c|c|c|c|c|}
\hline $\begin{array}{c}\text { Sample } \\
\text { (Interval in } \mathrm{cm} \text { ) }\end{array}$ & Spore Species Bases & Dinoflagellate Species Bases & Dinoflagellate Species Tops & Common Species \\
\hline $52-3,94-99$ & $\begin{array}{l}\text { Callialasporites trilobatus } \\
\text { Contignisporites cooksonï } \\
\text { Cyathidites australis } \\
\text { Klukisporites foveolatus } \\
\text { Klukisporites sp. A (PI. 11, Fig. 11-12) } \\
\\
\text { Aequitriradites spimulosus } \\
\text { Callialoasporites dampieri } \\
\text { Cerebropollenites mesozoicus } \\
\text { Cicatricosisporites hallei } \\
\text { Concavissimisporites variverrucatus } \\
\text { Klukisporites pseudoreticulatus } \\
\text { Corollina torosus }\end{array}$ & $\begin{array}{l}\text { Ctenidodinium panneum } \\
\text { Cometodinium sp. A (Habib, 1972) } \\
\text { Gonyaulacysta granulata } \\
\text { Hystrichodinium sp. A (loannides et al., 1977) } \\
\text { Imbatodinium kondratjevi } \\
\text { Muderongia simplex } \\
\text { PProlixosphaeridium torynum } \\
\text { Systematophora fasciculigera } \\
\text { Systematophora sp. A (Habib, 1972) } \\
\text { Tenua hystrix } \\
\text { Gonyaulacysta fastigiata } \\
\text { Senoniasphaera cf. jurassica (Pl. 3, Fig. 4) } \\
\text { Tenua verrucosa (sensu Habib, 1972) }\end{array}$ & $\begin{array}{l}\text { Ctenidodinium penneum } \\
\text { Imbatodinium kondratievi } \\
\text { ?Prolixosphaeridium torynum } \\
\text { Senoniasphaera sp. cf. S. jurassica } \\
\text { Systematophora fasciculigera }\end{array}$ & $\begin{array}{l}\text { Gonyaulacysta gramulate } \\
\text { ? Prolixosphaeridium torynum } \\
\text { Senoniasphaera sp. cf. S. jurassica }\end{array}$ \\
\hline
\end{tabular}

TABLE 2

Details of Spore and Dinoflagellate Species Occurrences in the Berriasian Sequence, Site 416

\begin{tabular}{|c|c|c|c|c|c|}
\hline $\begin{array}{c}\text { Sample } \\
\text { (Interval in } \mathrm{cm} \text { ) }\end{array}$ & Spore Species Bases & Spore Species Tops & Dinoflagellate Species Bases & $\begin{array}{l}\text { Dinoflagellate } \\
\text { Species Tops }\end{array}$ & Common Species \\
\hline $\begin{array}{l}416 A-32-5,11-15 \\
34 \times 1,63-67 \\
34-3,24-28\end{array}$ & \multicolumn{5}{|c|}{$\begin{array}{l}\text { (NO ADDITIONAL SPECIES) } \\
\text { (NO ADDITIONAL SPECIES) } \\
\text { (NO ADDITIONAL SPECIES) }\end{array}$} \\
\hline $\begin{array}{l}35-1,69-73 \\
35-3,70-74 \\
\end{array}$ & Triletes sp. A (Pl. 11, Fig. 4) & & $\begin{array}{l}\text { Phoberocysta neocomica } \\
\text { Trichodinium ciliatum }\end{array}$ & Trichodinium ciliatum & \\
\hline $\begin{array}{l}36-1,124-128 \\
37-1,80-84 \\
37-3,101-105\end{array}$ & $\begin{array}{l}\text { Foraminisporites wonthaggiensis } \\
\text { Perinopollenites elatoides }\end{array}$ & & $\begin{array}{l}\text { Muderongia sp. cf. } M \text {. simplex (PI. 5, Fig. 3,5) } \\
\text { Cyclonephelium distinctum } \\
\text { Cribroperidinium sp. A (PI. 2, Fig. 8-9) } \\
\text { Muderongia perforata } \\
\text { Kleithriasphaeridium eoinoides }\end{array}$ & & \\
\hline $\begin{array}{l}38-1,78-82 \\
40-1,104-108\end{array}$ & \multicolumn{5}{|c|}{$\begin{array}{l}\text { (NO ADDITIONAL SPECIES) } \\
\text { (BARREN) }\end{array}$} \\
\hline $\begin{array}{l}40-3,60-64 \\
40-5,79-83\end{array}$ & \multicolumn{5}{|c|}{$\begin{array}{c}\text { (NO ADDITIONAL SPECIES) } \\
\text { (BARREN) }\end{array}$} \\
\hline $\begin{array}{l}41-3,5-9 \\
41-4,70-75\end{array}$ & $\begin{array}{l}\text { Deltoidospora psilostoma } \\
\text { Rouseisporites reticulatus } \\
\text { Aequitriradites verrucosus } \\
\text { Appendicisporites jansonii }\end{array}$ & Klukisporites sp. A & $\begin{array}{l}\text { Achomosphaera neptuni } \\
\text { Cyclonephelium distinctum subsp. brevispinatum } \\
\text { Systematophora sp. cf. S. areolata ( } \mathrm{Pl} .4 \text {. Fig. } \\
\text { 4: Pl. 7, Fig. 6) } \\
\text { Hystrichosphaeridium recurvatum }\end{array}$ & & \\
\hline $\begin{array}{l}42-1,75-79 \\
42-3,98-102 \\
43-1,104-108\end{array}$ & \multicolumn{5}{|c|}{$\begin{array}{l}(\text { BARREN) } \\
(\text { BARREN) } \\
(\text { BARREN) }\end{array}$} \\
\hline $\begin{array}{l}43-3,91-94 \\
44-1,43-47\end{array}$ & $\begin{array}{l}\text { Trilobosporites apiverrucatus } \\
\text { Callialasporites segmentatus } \\
\text { Cicatricosisporites hughesi } \\
\text { Trilobosporites jurassicus } \\
\text { Verrucosisporites rotundus }\end{array}$ & & $\begin{array}{l}\text { Muderongia tetracantha } \\
\text { Ctenidodinium culmulum } \\
\text { Endoscrinium campanulum } \\
\text { Polysphaeridium sp. A (PI. 1. Fig. 2; P1. 8, } \\
\text { Fig. 2, 4-5) }\end{array}$ & Muderongia tetracantha & $\begin{array}{l}\text { Muderongia simplex } \\
\text { Muderongia tetracantha } \\
\text { Muderongia simplex } \\
\text { Verrucosisporites spp. }\end{array}$ \\
\hline $\begin{array}{l}45-1,87-92 \\
45-3,45-49 \\
46-1,32-36 \\
46-3,114-118 \\
47-1,57-61 \\
\end{array}$ & \multicolumn{5}{|c|}{$\begin{array}{l}\text { (BARREN) } \\
\text { (BARREN) } \\
\text { (BARREN) } \\
\text { (BARREN) } \\
(\text { BARREN) } \\
\end{array}$} \\
\hline $\begin{array}{l}48-3,129-132 \\
49-1,134-137\end{array}$ & \multicolumn{5}{|c|}{$\begin{array}{l}\text { (NO ADDITIONAL SPECIES) } \\
\text { (BARREN) }\end{array}$} \\
\hline $50-1,45-49$ & $\begin{array}{l}\text { Acanthotriletes varispinosus } \\
\text { Cicatricosisporites augustus } \\
\text { Cicatricosisporites australiensis } \\
\text { Cicatricososporites auritus } \\
\text { Leptolepidites psarosus }\end{array}$ & & $\begin{array}{l}\text { Pyxidiella sp. A (Habib, 1972) } \\
\text { Scriniocassis dictyotus (sensu Habib, 1972) } \\
\text { Dingodinium cerviculum } \\
\text { Tanyosphaeridium boletum }\end{array}$ & & \\
\hline $50-3.58-62$ & \multicolumn{5}{|c|}{ (BARREN) } \\
\hline
\end{tabular}


TABLE 3

Details of Spore and Dinoflagellate Species Occurrences in the Valanginian Sequence, Site 416

\begin{tabular}{|c|c|c|c|c|c|}
\hline $\begin{array}{c}\text { Sample } \\
\text { (Interval in cm) }\end{array}$ & Spore Species Bases & Spore Species Tops & Dinoflagellate Species Bases & Dinoflagellate Species Tops & Common Species \\
\hline $416 \mathrm{~A}-10-1,83-86$ & & $\begin{array}{l}\text { Appendicisporites jansonii } \\
\text { Cicatricosisporites augustus } \\
\text { Cicatricosisporites australiensis }\end{array}$ & & Muderongia simplex & \\
\hline $11-1,64-68$ & & $\begin{array}{l}\text { Aequitriradites spinulosus } \\
\text { Appendicisporites bilateralis } \\
\text { Appendicisporites concentricus } \\
\text { Cyathiditites australis }\end{array}$ & & & \\
\hline $11-3,78-81$ & & Cicatricosisporites subrotundus & & $\begin{array}{l}\text { Muderongia perforata } \\
\text { Prolixospheeridium xanthiopyxides } \\
\text { Spiniferites speciosus }\end{array}$ & \\
\hline $11-5,19-23$ & & $\begin{array}{l}\text { Densoisporites velatus } \\
\text { Klukisporites pseudoreticulatus } \\
\text { Rouseisporites reticulatus }\end{array}$ & & Endoscrinium campanulum & \\
\hline $12-1,112-115$ & & $\begin{array}{l}\text { Concavissimisporites variverrucatus } \\
\text { Leptolepidites psarosus }\end{array}$ & & Tenua verrucosa (sensu Habib, 1972) & \\
\hline $12-2,62-66$ & & & & $\begin{array}{l}\text { Cribroperidinium sepimentum } \\
\text { Diacanthum hollisteri } \\
\text { Perisseisphaeridium sp. A } \\
\text { Perisseiasphaeridium sp. B }\end{array}$ & \\
\hline $12-4,112-116$ & Trilobosporites trioreticulosus & $\begin{array}{l}\text { Contignisporites cooksonii } \\
\text { Pilosisporites sp. A }\end{array}$ & & $\begin{array}{l}\text { Gonyaulacysta granulata } \\
\text { Tenua hystrix }\end{array}$ & Cicatricosisporites hallei \\
\hline $13-1,138-141$ & Appendicisporites bilateralis & & & & \\
\hline $14-1,89.91$ & & $\begin{array}{l}\text { Callialasporites segmentatus } \\
\text { Cyathidites minor } \\
\text { Trilobosporites apiverrucatus }\end{array}$ & & Hystrichodinium pulchrum & \\
\hline $14-3,143-145$ & & Deltoidospora psilostoma & & & Cicatricosisporites australiensis \\
\hline $14-5,41-42$ & Pilosisporites trichopapillosus & $\begin{array}{l}\text { Pilosisporites trichopapillosus } \\
\text { Trilobosporites purvermilentus }\end{array}$ & $\begin{array}{l}\text { Prolixosphaeridium xanthiopyxides } \\
\text { Trilobosporites purverrulentus }\end{array}$ & & \\
\hline $15-1,41-45$ & & & & $\begin{array}{l}\text { Kleithriasphaeridium fasciatum } \\
\text { Systematophora fasciculigera (sensu Habib, 1972) }\end{array}$ & \\
\hline $15-3,54-58$ & & & & $\begin{array}{l}\text { Cribroperidinium sp. A } \\
\text { Systematophora complicata } \\
\text { Systematophora fasciculigera } \\
\text { Systematophora schindewolfii }\end{array}$ & \\
\hline $\begin{array}{l}15-5,79-82 \\
16-2,91-92 \\
\end{array}$ & & & $\begin{array}{l}\text { (NO ADDITIONAL SP } \\
\text { (NO ADDITIONAL SP) } \\
\end{array}$ & & \\
\hline $16-4,55-59$ & & $\begin{array}{l}\text { Callialasporites trilobatus } \\
\text { Costatoperforosporites foveolatus }\end{array}$ & $\begin{array}{l}\text { Oligosphaeridium complex } \\
\text { Oligosphaeridium sp. A, PL. 4, fig. 1-2 }\end{array}$ & $\begin{array}{l}\text { Cyclonephelium distinctum subsp. brevispinatum } \\
\text { Oligosphaeridium complex } \\
\text { oligosphaeridium sp. A }\end{array}$ & Cyclonephelium distinctum \\
\hline $17-1,65-69$ & Costatoperforosporites foveolatus & $\begin{array}{l}\text { Cicatricososporites auritus } \\
\text { Forminisporis wonthaggiensis } \\
\text { Trilobosporites bernissartensis } \\
\text { Tritobosporites jurassicus } \\
\text { Perinopollenites elatooides }\end{array}$ & & & \\
\hline $17-3,122-124$ & & $\begin{array}{l}\text { Aequitriradites verrucosus } \\
\text { Verrucosisporites rotundus }\end{array}$ & & & \\
\hline $18-1,134-138$ & & Cicatricosisporites hughesi & & & \\
\hline $18-4,40-43$ & & Cicatricosisporites dorogensis & & Ctenidodinium culmulum & \\
\hline $19-1,142-148$ & & & (NO ADDITIONAL SP & & \\
\hline $19-3,36-39$ & & $\begin{array}{l}\text { Acanthotriletes varispinosus } \\
\text { Ephedripites sp. A A } \\
\text { Klukisporites foveolatus }\end{array}$ & & $\begin{array}{l}\text { Dingodinium cerviculum } \\
\text { Pyxidiella sp. A (Habib, 1972) } \\
\text { Tenaa anphrissa }\end{array}$ & $\begin{array}{l}\text { Cyclonephelium distinctum subsp. brevispinatum } \\
\text { Pseudoceratium pelliferum }\end{array}$ \\
\hline $19-5,82-86$ & Pilosisporites sp. A & Laricoidites magnus & & & \\
\hline $20-1,21-25$ & & & & Polysphaeridium sp. A & \\
\hline $21-1,66-69$ & & Lycopodiumsporites crassimacerius & Canningia colliveri & & \\
\hline $21-3,121-125$ & & & (NO ADDITIONAL SP & & \\
\hline $21-5,17-21$ & & & Cribroperidinium orthoceras & $\begin{array}{l}\text { Cribroperidinium orthoceras } \\
\text { Muderongia sp. cf. M. simplex }\end{array}$ & \\
\hline $22-1,128-132$ & Trilobosporites purvernulensis & & & & \\
\hline
\end{tabular}




\begin{tabular}{|c|c|c|c|c|c|}
\hline \multirow{2}{*}{$\frac{22-3,38-41}{22-5,51-54}$} & \multicolumn{5}{|c|}{ (NO ADDITIONAL SPECIES) } \\
\hline & \multicolumn{5}{|c|}{ (NO ADDITIONAL SPECIES) } \\
\hline $23-1,107 \cdot 111$ & & & Cyclonephelium vannophorum & Hystrichodinium sp. A (loannides et al., 1977) & \\
\hline $23-2,67-71$ & & & & Gonyaulacysta fastigiata & \\
\hline $23-4,72-77$ & & & & Hystrichosphaeridium recurvatum & \\
\hline $24-1,90-95$ & $\begin{array}{l}\text { Cyathidites minor } \\
\text { Densoisporites velatus }\end{array}$ & & & & Pseudoceratium pelliferum \\
\hline $24-3,57-61$ & & Leptolepidites verrucatus & & $\begin{array}{l}\text { Canningia sp. A } \\
\text { Oligosphaeridium perforatum } \\
\text { Scriniocassis dictyotus (sensu Habib, 1972) }\end{array}$ & $\begin{array}{l}\text { Canningia sp. A } \\
\text { Ephedripites sp. A }\end{array}$ \\
\hline $25-1,60-66$ & & & & & Pseudoceratium pellifenum \\
\hline $25-3,90-94$ & & & $\begin{array}{l}\text { Canningia sp. A (P1.6, Fig. 5-6) } \\
\text { Oligosphoeridium perforatum } \\
\text { Perisseiasphaeridium sp. B (Pl. 4, Fig. 12) }\end{array}$ & Kalyptea monoceras & Pseudoceratium pelliferum \\
\hline $26-1,98-102$ & & & Systematophora complicata & & \\
\hline $26-3,50-53$ & \multicolumn{5}{|c|}{ (NO ADDITIONAL SPECIES) } \\
\hline $26-5,88-92$ & & & & Tenua sp. A & \\
\hline $27-1,64-67$ & \multicolumn{5}{|c|}{ (NO ADDITIONAL SPECIES) } \\
\hline $27-3,7-11$ & & & Tenue sp. A (PI, 6, Fig. 1,2) & & \\
\hline $27-5,121-125$ & \multicolumn{5}{|c|}{ (NO ADDITIONAL SPECIES) } \\
\hline $28-1,30-34$ & Distaltriangulisporites perplexus & Distaltriangulisporites perplexus & & $\begin{array}{l}\text { Leptodinium sp. A } \\
\text { Prolixosphaeriditum mixtispinosum } \\
\text { Systematophora sp. cf. } S \text {. areolata }\end{array}$ & $\begin{array}{l}\text { Prolixosphaeridium mixtispinosum } \\
\text { Pseudoceratium pelliferum }\end{array}$ \\
\hline $28-3,58-62$ & $\begin{array}{l}\text { Qcictricosisporites dorogensis } \\
\text { Trilobosporites bernissartensis }\end{array}$ & Cerebropollenites masozoicus & Systematophora schindewolfii & & \\
\hline $28-5,27-30$ & & & & Systematophora sp. A (Habib, 1972) & \\
\hline $29-1,18-22$ & $\begin{array}{l}\text { Appendicisporites concentricus } \\
\text { Laricoidites magnus }\end{array}$ & & & Kallosphaeridium sp. A & \\
\hline $29-4,142-146$ & \multicolumn{5}{|c|}{ (NO ADDITIONAL SPECIES) } \\
\hline $29-6,57-61$ & & & $\begin{array}{l}\text { Kleithriasphaeridium fasciatum } \\
\text { Perisseiasphaeridium sp. A (P1. 4, Fig. 10) }\end{array}$ & & \\
\hline $30-1,99-103$ & Leptolepidites verrucatus & & $\begin{array}{l}\text { Cribroperidinium sepimentum } \\
\text { Temua anaphrissa } \\
\text { Wallodinium knutzschi }\end{array}$ & $\begin{array}{l}\text { Achomosphaera neptuni } \\
\text { Chlamydophorella sp. A } \\
\text { Ctenidodinizum elegantulum } \\
\text { Wallodinium krutzschi }\end{array}$ & \\
\hline $30-3,46-49$ & $\begin{array}{l}\text { Cicatricosisporites subrotundus } \\
\text { Lycopodiumsporites crassi- } \\
\text { macerius }\end{array}$ & Triletes sp. A & & & \\
\hline $30-5,77-81$ & \multicolumn{5}{|c|}{ (NO ADDITIONAL SPECIES) } \\
\hline $31-1,15-19$ & & & & & Klukisporites pseudoreticulatus \\
\hline $31-3,70-74$ & & & $\begin{array}{l}\text { Crenidodinium elegantulum } \\
\text { Hystrichodinium pulchrum } \\
\text { Kalyptea monoceras } \\
\text { Leptodinium sp. A (P1. 2, Fig. 7) } \\
\text { Polysphaeridium warrenit } \\
\text { Prolixosphaeridium mixtispinosum }\end{array}$ & & 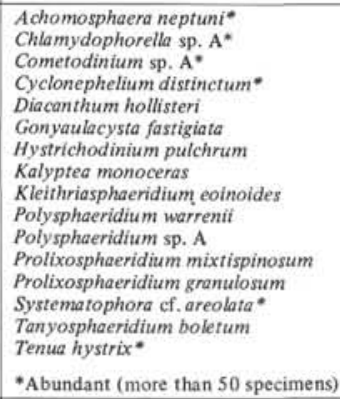 \\
\hline $31-5,80-84$ & & & Kallosphaeridium sp. A (P1. 3, Fig. 3) & & \\
\hline $32-1,27-31$ & \multicolumn{5}{|c|}{ (BARREN) } \\
\hline $32-3,125-129$ & & & \begin{tabular}{|l|} 
Chlamydophorella sp. A (PI. 3, Fig. 8,9) \\
Discanthum hollister \\
Prolixosphoeridium granulosum \\
Pseudoceratium pelliferum \\
Spiniferites speciosus \\
Systematophora fasciculigera (sensu Habib, 1972)
\end{tabular} & & \\
\hline
\end{tabular}


TABLE 4

Details of Spore and Dinoflagellate Occurrences in the Hauterivian Sequence, Sample 416A-9-4, 31-35

\begin{tabular}{|c|c|c|}
\hline Spore Species Tops & Dinoflagellate Species Bases & Dinoflagellate Species Tops \\
\hline $\begin{array}{l}\text { Callialasporites dampieri } \\
\text { Cicatricosisporites hallet } \\
\text { Corollina torosus } \\
\text { Trilobosporites trioreticulosus }\end{array}$ & $\begin{array}{l}\text { Cyclonephelium tabulatum } \\
\text { Meiourogonyaulax stoveri } \\
\text { Oligosphaeridium pulcherrimum }\end{array}$ & $\begin{array}{l}\text { Canningia colliveri } \\
\text { Cometodinium sp. A (Habib, 1972) } \\
\text { Cyclonephelium distinctum } \\
\text { Cyclonephelitum tabulatum } \\
\text { Cyclonephelium vannophorum } \\
\text { Kleithriasphaeridium eoinodes } \\
\text { Meiourogonyaulax stoveri } \\
\text { Oligosphaeridium pulcherrimum } \\
\text { Phoberocysta neocomica } \\
\text { Polysphaeridium multispinosum } \\
\text { Polysphaeridium narrenï } \\
\text { Prolixasphaeridium granulosum } \\
\text { Preudoceratium pelifferum } \\
\text { Tanyospheridium belotum }\end{array}$ \\
\hline
\end{tabular}

al., Initial Reports of the Deep Sea Drilling Project, v. 11: Washington.

Ioannides, N. S., Stavrinos, G. N., and Downie, C., 1977. Kimmeridgian microplankton from Clavel's Hard, Dorset, England, Micropaleontology, v. 22, p. 443-478.

Norris, G., 1965. Archeopyle structures in Upper Jurassic dinoflagellates from southern England, New Zealand Journal of Geological Geophysics, v. 8, p. 792-806.

Williams, G. L., 1978. Palynological biostratigraphy, Deep Sea Drilling Project Sites 367 and 370. In Supplement to Volumes 38, 39, and 40: Washington (U.S. Government Printing Office), p. 783-816.

\section{APPENDIX A}

Alphabetic listings of dinoflagellate and spore species recorded in this study. Plate and figure numbers are given for all illustrated taxa.

Acanthotriletes varispinosus Pocock.

Achomosphaera neptuni (Eisenack) Davey and Williams. Plate 5, Figures 7, 8. Plate 9, Figure 3.

Aequitriradites spinulosus (Cookson and Dettmann) Cookson and Dettmann.

Aequitriradites verrucosus (Cookson and Dettmann) Cookson and Dettmann. Plate 10, Figures 1-3.

Amphorula metaelliptica Dodekova. Plate 3, Figure 10.

Appendicisporites bilateralis Singh.

Appendicisporites concentricus Kemp. Plate 10, Figure 11.

Appendicisporites jansonii Pocock.

Callialasporites dampieri (Balme) Dev.

Callialasporites segmentatus (Balme) Dev.

Callialasporites trilobatus (Balme) Dev. Plate 11, Figure 6.

Canningia colliveri Cookson and Eisenack.

Canningia sp. A. Plate 6, Figures 5, 6.

Cerebropollenites mesozoicus (Couper) Nilsson.

Chlamydophorella sp. A. Plate 3, Figures 8, 9.

Cicatricosisporites augustus Singh.

Cicatricosisporites australiensis (Cookson) Potonié.

Cicatricosisporites dorogensis Potonié and Gelletich.

Cicatricosisporites hallei Delcourt and Sprumont.

Cicatricosisporites hughesi Dettmann. Plate 10, Figures 4, 5 .

Cicatricosisporites subrotundus Brenner.

Cicatricososporites auritus Singh. Plate 10, Figures 8, 9.

Cometodinium sp. A (Habib, 1972). Plate 3, Figures 11, 12; Plate 9, Figures 4, 6 .

Concavissimisporites variverrucatus (Couper) Brenner.

Contignisporites cooksonii (Balme) Dettmann. Plate 10, Figure 10. Corollina torosus (Reissinger) Klaus.

Costatoperforosporites foveolatus Deák.

Cribroperidinium orthoceras (Eisenack) Davey.

Cribroperidinium sepimentum Neale and Sarjeant.

Cribroperidinium sp. A. Plate 2, Figures 8, 9.

Ctenidodinium culmulum (Norris) Lentin and Williams.

Ctenidodinium elegantulum Millioud. Plate 3, Figure 6.

Ctenidodinium panneum (Norris) Lentin and Williams.
Cyathidites australis Couper.

Cyathidites minor Couper.

Cyclonephelium distinctum Deflandre and Cookson. Plate 7, Figures $1,4$.

Cyclonephelium distinctum subsp. brevispinatum (Millioud) Lentin and Williams. Plate 6, Figures 3, 4.

Cyclonephelium tabulatum Davey and Verdier.

Cyclonephelium vannophorum Davey.

Deltoidospora psilostoma Rouse.

Densoisporites velatus Weyland and Krieger.

Diacanthum hollisteri Habib.

Dingodinium cerviculum Cookson and Eisenack. Plate 5, Figure 6.

Distaltriangulisporites perplexus (Singh) Singh.

Endoscrinium campanulum (Gocht) Vozzhennikova.

Ephedripites sp. A. Plate 10, Figure 7.

Foraminisporis wonthaggiensis (Cookson and Dettmann) Dettmann.

Gonyaulacysta fastigiata Duxbury. Plate 9, Figures 1, 2.

Gonyaulacysta granulata (Klement) Sarjeant.

Hystrichodinium pulchrum Deflandre.

Hystrichodinium sp. A (Ioannides et al., 1977). Plate 5, Figures 9, 10.

Hystrichosphaeridium recurvatum (White) Davey and Williams. Plate 1, Figure 12.

Imbatodinium kondratjevii Vozzhennikova. Plate 2, Figure 4.

Kallosphaeridium sp. A. Plate 3, Figure 3.

Kalyptea monoceras Cookson and Eisenack. Plate 5, Figure 11.

Kleithriasphaeridium eoinodes (Eisenack) Davey. Plate 4, Figures 3-9.

Kleithriasphaeridium fasciatum (Davey and Williams) Davey.

Klukisporites foveolatus Pocock.

Klukisporites pseudoreticulatus Couper.

Klukisporites sp. A. Plate 11, Figures 11, 12.

Earicoidites magnus (Potonié) Potonié, Thomson and Thiergart.

Leptodinium sp. A. Plate 2, Figure 7.

Leptolepidites psarosus Norris. Plate 11, Figure 9.

Leptolepidites verrucatus Couper. Plate 11, Figure 7.

Lycopodiumsporites crassimacerius Hedlund.

Meiourogonyaulax stoveri Millioud.

Muderongia perforata Alberti.

Muderongia simplex Alberti. Plate 5, Figures 2, 4.

Muderongia sp. cf. M. simplex. Plate 5, Figures 3, 5.

Muderongia tetracantha (Gocht) Alberti.

Oligosphaeridium complex (White) Davey and Williams.

Oligosphaeridium perforatum (Gocht) Davey and Williams.

Oligosphaeridium pulcherrimum (Deflandre and Cookson) Davey and Williams.

Oligosphaeridium sp. A. Plate 4, Figures 1, 2.

Perinopollenites elatoides Couper.

Perisseiasphaeridium sp. A. Plate 4, Figure 10.

Perisseiasphaeridium sp. B. Plate 4, Figure 12.

Phoberocysta neocomica (Gocht) Millioud.

Pilosisporites trichopapillosus (Thiergart) Delcourt and Sprumont.

Pilosisporites sp. A. Plate 11, Figures 1-3.

Polysphaeridium multispinosum Davey. Plate 1, Figures 3, 4; Plate 8, Figure 1.

Polysphaeridium warrenii Habib. Plate 1, Figure 1.

Polysphaeridium sp. A. Plate 1, Figure 2; Plate 8, Figures 2, 4, 5.

Prolixosphaeridium granulosum (Deflandre) Davey et al. Plate 1, Figure 11; Plate 8, Figure 6.

Prolixosphaeridium mixtispinosum (Klement) Davey et al.

?Prolixosphaeridium torynum (Cookson and Eisenack) Eisenack and Kjellström. Plate 3, Figure 7.

?Prolixosphaeridium xanthiopyxides (O. Wetzel) Davey et al.

Pseudoceratium pelliferum Gocht. Plate 2, Figures 1-3; Plate 9, Figure 5.

Pyxidiella sp. A. (Habib, 1972). Plate 2, Figure 6.

Rouseisporites reticulatus Pocock. Plate 11, Figure 10.

Scriniocassis dictyotus (Cookson and Eisenack) Beju (sensu Habib, 1972). Plate 3, Figures 1, 2.

Senoniasphaera sp. cf. S. jurassica (Gitmez and Sarjeant) Lentin and Williams. Plate 3, Figure 4.

?Spiniferites dentatus (Gocht) Lentin and Williams.

Spiniferites speciosus (Deflandre) Sarjeant.

Systematophora sp. cf. S. areolota Klement. Plate 4, Figure 4; Plate 7, Figure 6. 
Systematophora complicata Neale and Sarjeant.

Systematophora fasciculigera Klement. Plate 4, Figure 7.

Systematophora fasciculigera Klement (sensu Habib, 1972).

Systematophora schindewolfii (Alberti) Downie and Sarjeant. Plate 4, Figures 5, 6.

Systematophora sp. A (Habib, 1972). Plate 4, Figure 8.

Tanyosphaeridium boletum Davey. Plate 1, Figures 5-9; Plate 8, Figure 3.

Tenua anaphrissa (Sarjeant) Benedek.

Tenua hystrix Eisenack. Plate 3, Figure 5; Plate 7, Figures 2, 5.

Tenua verrucosa Sarjeant (sensu Habib, 1972).
Tenua sp. A. Plate 6, Figures 1, 2.

Trichodinium ciliatum Gocht.

Trilobosporites apiverrucatus Couper.

Trilobosporites bernissartensis (Delcourt and Sprumont) Potonié.

Trilobosporites jurassicus Pocock. Plate 11, Figure 5.

Trilobosporites purverulentus (Verbitskaya) Dettmann.

Trilobosporites trioreticulosus Cookson and Dettmann.

Triletes sp. A. Plate 11, Figure 4.

Verrucosisporites rotundus Singh.

Wallodinium krutzschi (Alberti) Habib. Plate 2, Figure 5. 
PLATE 1

Bar on all figures equals $30 \mu \mathrm{m}$.

Figure 1 Polysphaeridium warrenii. Sample 18-4, 40-43 cm. GSC No. 56697.

Figure 2 Polysphaeridium sp. A. Sample 31-3, 70-74 cm. GSC No. 56698.

Figure 3 Polysphaeridium multispinosum. Sample 31-3, 70-74 cm. GSC No. 56699.

Figure 4 Polysphaeridium multispinosum. Sample 29-6, 57-61 cm. GSC No. 56700.

Figure 5 Tanyosphaeridium boletum. Sample 15-1, 41-45 cm. GSC No. 56701.

Figure 6 Tanyosphaeridium boletum. Sample 24-3, 57-61 cm. GSC No. 56702 .

Figure 7 Tanyosphaeridium boletum. Sample 27-1, 64-67 cm. GSC No. 56703.

Figures 8, 9 Tanyosphaeridium boletum. Sample 16-4, 55-59 cm. GSC No. 56704.

Figure 10 Specimen intermediate between Tanyosphaeridium boletum, Prolixosphaeridium granulosum and Polysphaeridium multispinosum. Sample 31-3, 70-74 cm. GSC No. 56705.

Figure 11 Prolixosphaeridium granulosum. Sample 31-3, 70-74 cm. GSC No. 56706.

Figure 12 Hystrichosphaeridium recurvatum. Sample 31-3, 70-74 cm. GSC No. 56707. 
PLATE 1
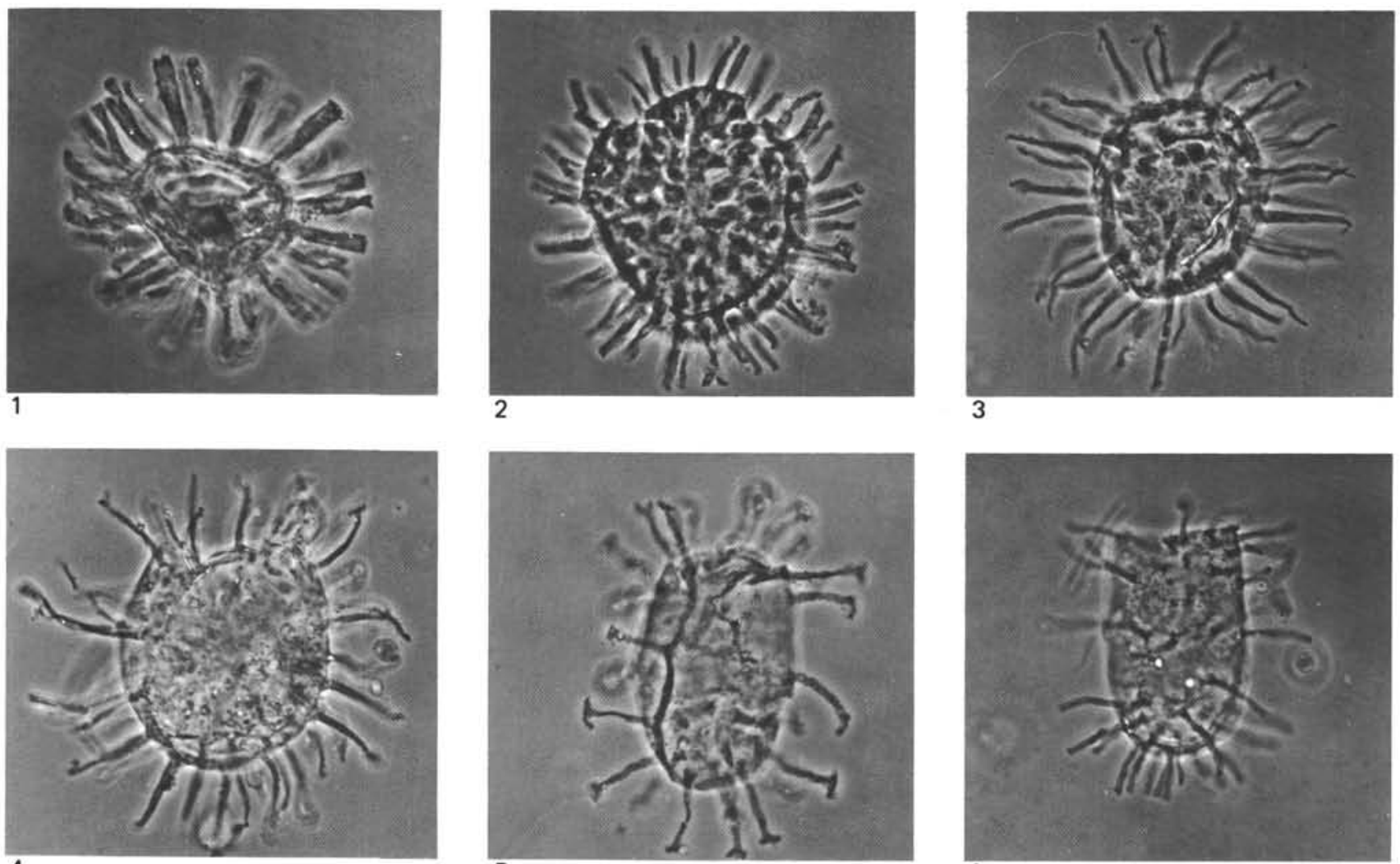

4

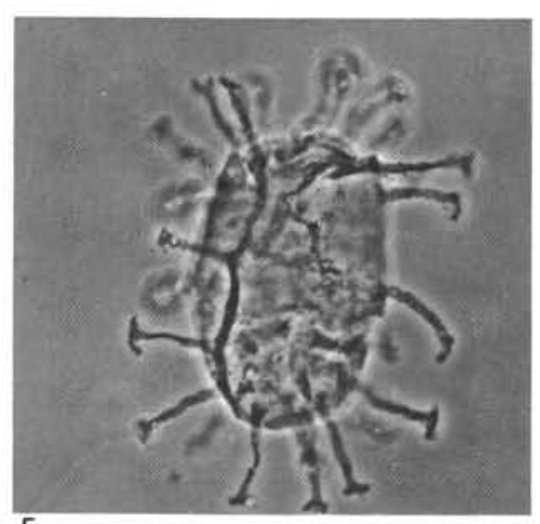

3
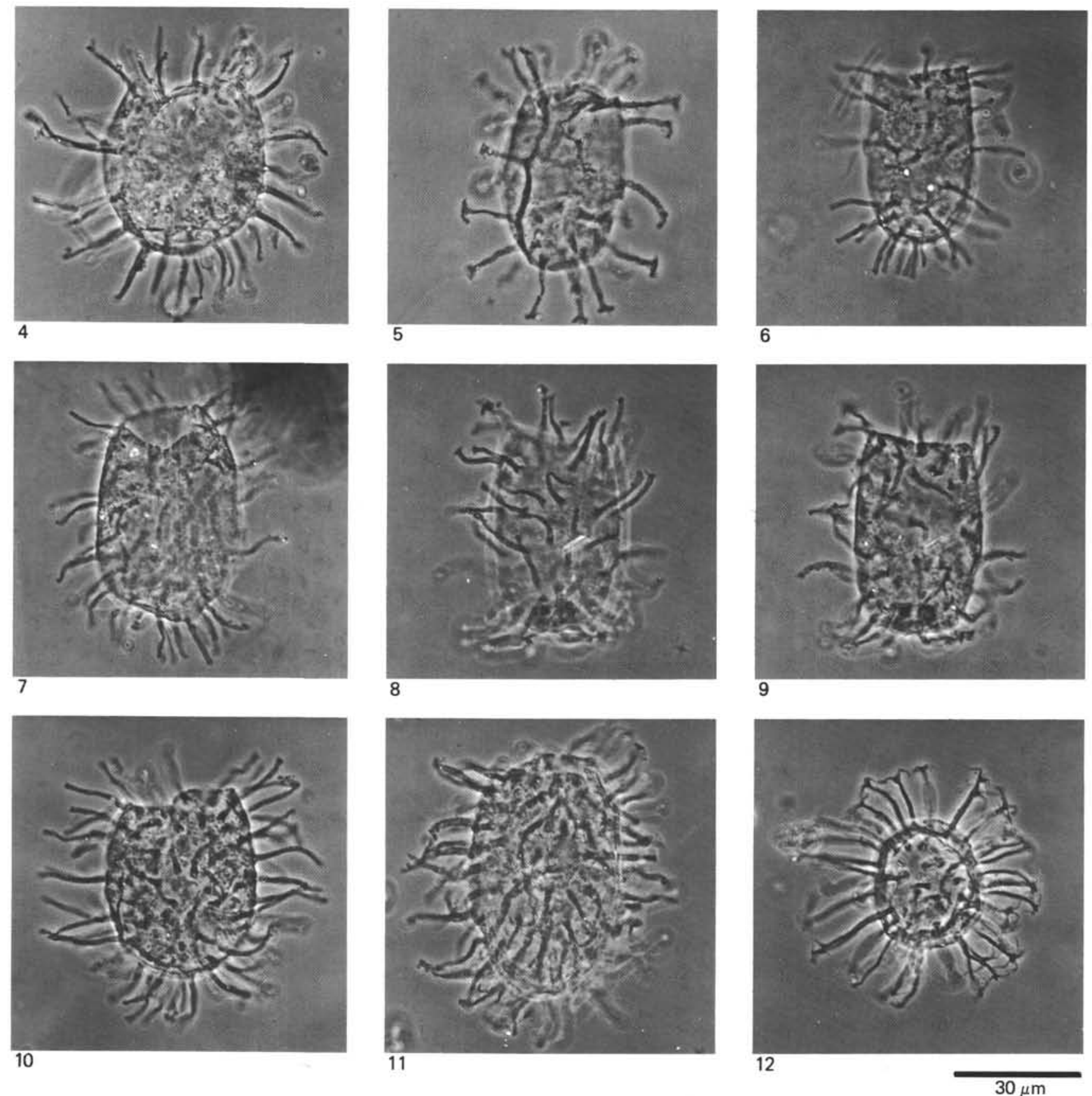


\section{PLATE 2}

Bar on all figures equals $30 \mu \mathrm{m}$.

Figure 1 Pseudoceratium pelliferum. Sample 14-3, 143-145 cm. GSC No. 56708.

Figure 2 Pseudoceratium pelliferum, form with strongly reduced postcingular horn. Sample 25-1, 60-66 cm. GSC No. 56709.

Figure 3 Pseudoceratium pelliferum, form with reduced postcingular horn. Sample $25-1,60-66 \mathrm{~cm}$. GSC No. 56710 .

Figure 4 Imbatodinium kondratjevii, form with ?2 intercalaries removed. Sample 51-1, 17-21 cm. GSC No. 56711 .

Figure 5 Wallodinium krutzschi. Sample 30-1, 99-103 cm. GSC No. 56712.

Figure 6 Pyxidiella sp. A (Habib, 1972). Sample 19-3, $36-39 \mathrm{~cm}$. GSC No. 56713.

Figure $7 \quad$ Leptodinium $\mathrm{sp}$. A. Sample 28-1, 30-34 cm. GSC No. 56714.

Figure 8 Cribroperidinium sp. A. Sample 24-3, 57-61 cm. GSC No. 56715.

Figure 9 Cribroperidinium sp. A. Sample 51-1, 17-21 cm. GSC No. 56716. 
PLATE 2
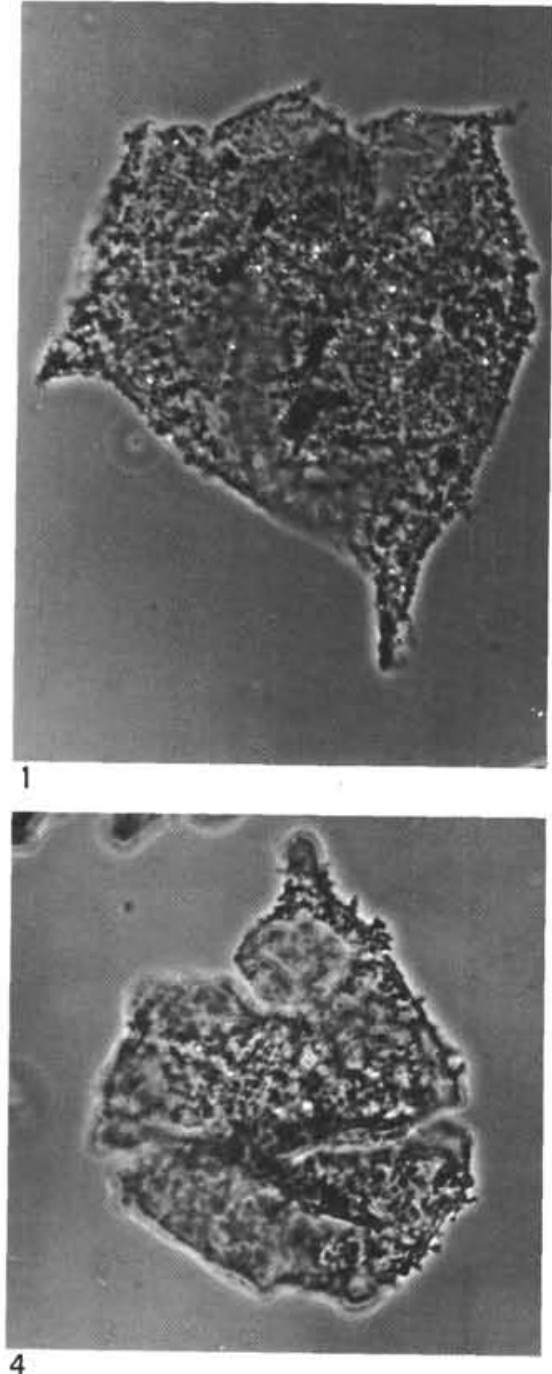

$$
4
$$

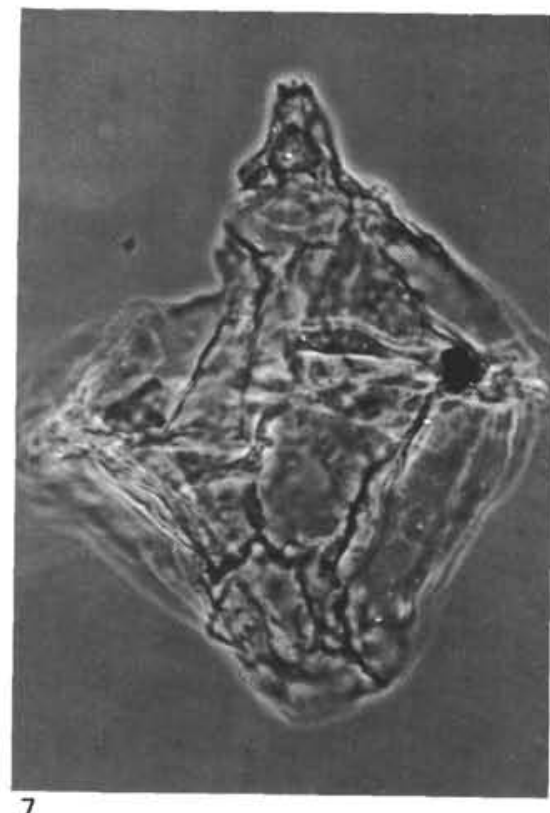

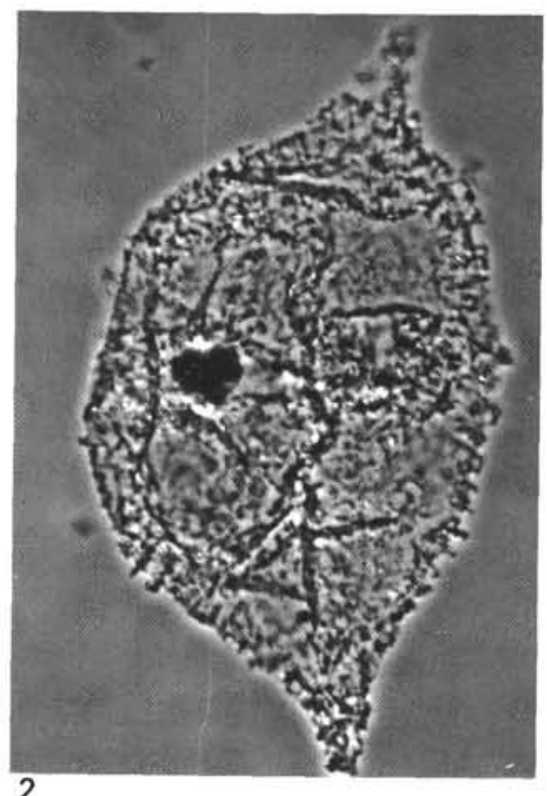
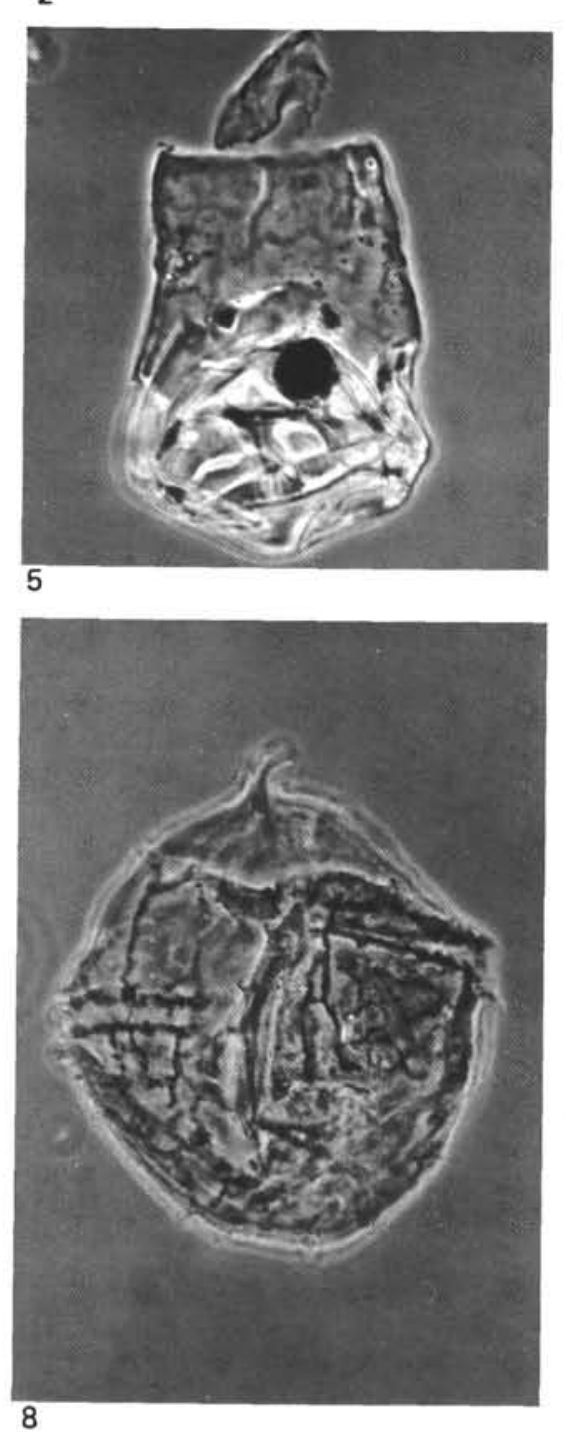

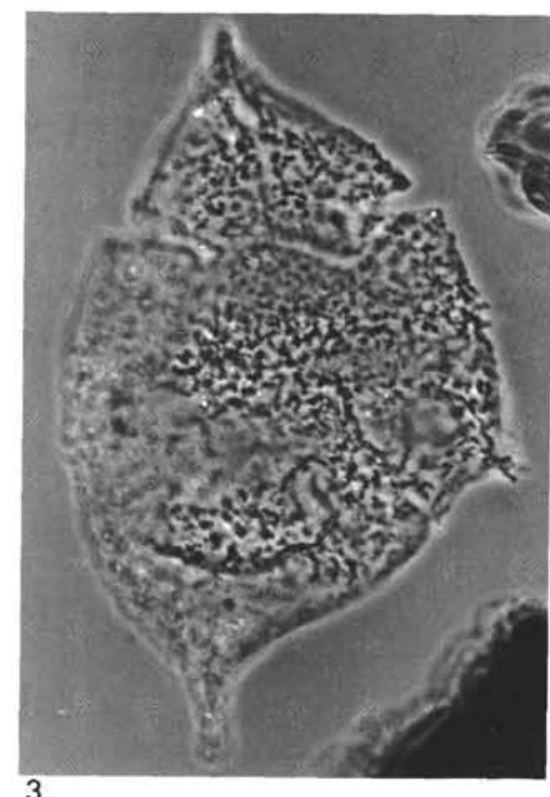

3
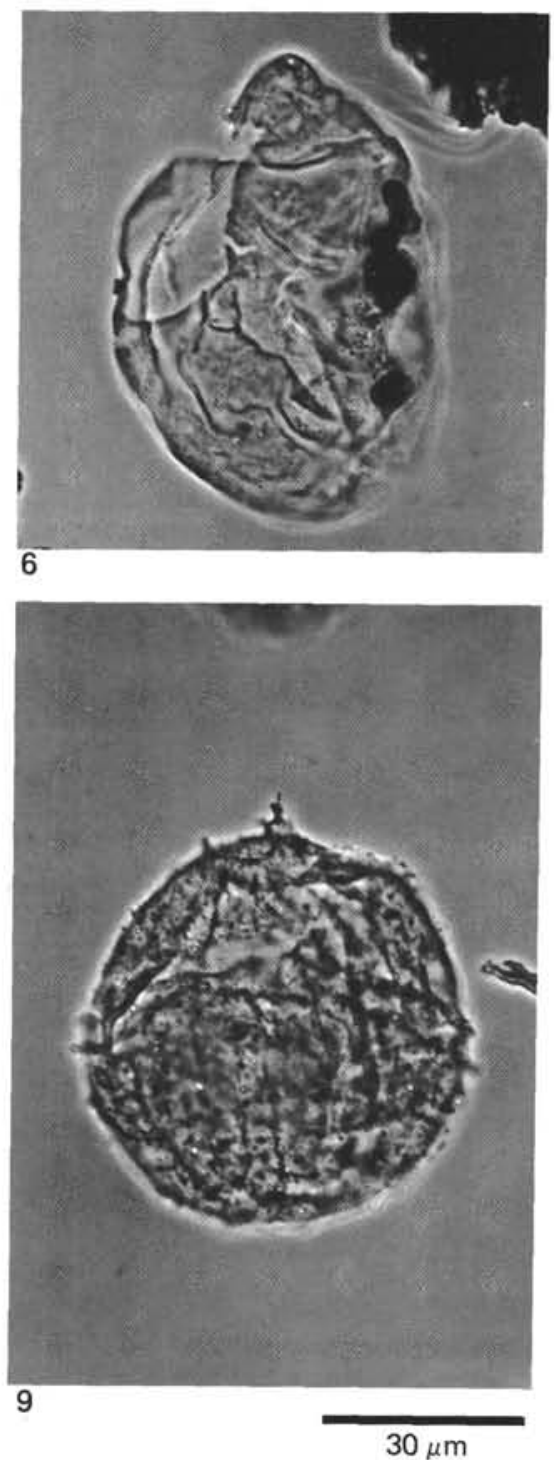


\section{PLATE 3}

Bar on all figures equals $30 \mu \mathrm{m}$.

Figures 1, 2 Scriniocassis dictyotus. Sample 41-4, 70-75 cm. GSC No. 56717.

Figure 3 Kallosphaeridium sp. A. Sample 29-1, 18-22 cm. GSC No. 56718.

Figure $4 \quad$ Senoniasphaera sp. cf. S. jurassica. Sample 51-1, 17-21 cm. GSC No. 56719.

Figure 5 Tenua hystrix. Sample 31-3, 70-74 cm. GSC No. 56720.

Figure 6 Ctenidodinium elegantulum. Sample 30-1, 99-103 cm. GSC No. 56721.

Figure 7 ?Prolixosphaeridium torynum. Sample 51-1, 17-21 cm. GSC No. 56722 .

Figure $8 \quad$ Chlamydophorella sp. A. Sample 30-1, 99-103 $\mathrm{cm}$. GSC No. 56723.

Figure 9 Chlamydophorella sp. A. Sample 30-1, 99-103 cm. GSC No. 56724 .

Figure 10 Amphorula metaelliptica. Sample 51-1, 17-21 cm. GSC No. 56725.

Figure 11 Cometodinium sp. A (Habib, 1972). Sample 31-3, 70-74 cm. GSC No. 56726.

Figure 12 Cometodinium sp. A (Habib, 1972). Sample 30-5, $77-81 \mathrm{~cm}$. GSC No. 56727. 
PLATE 3
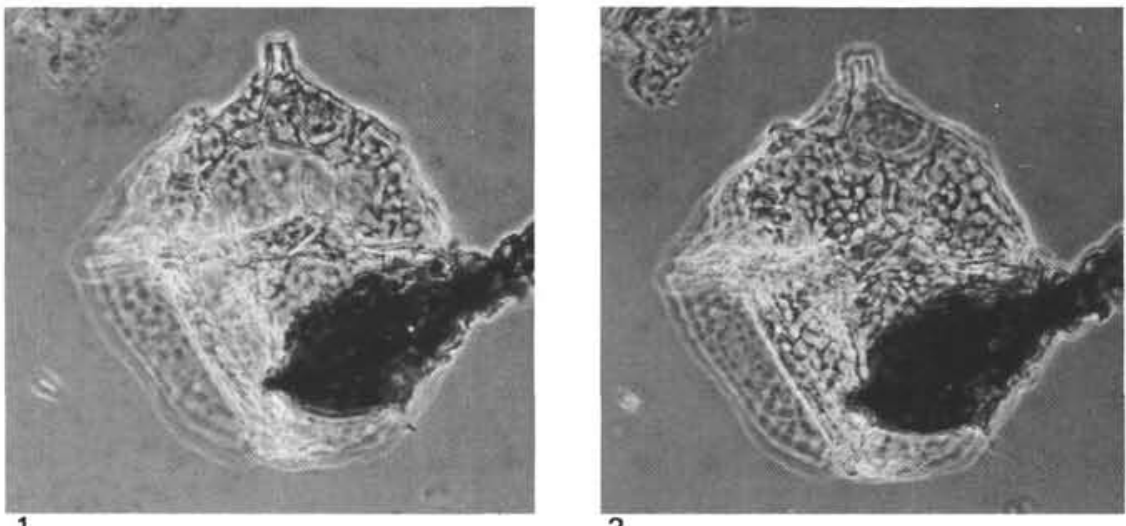

2

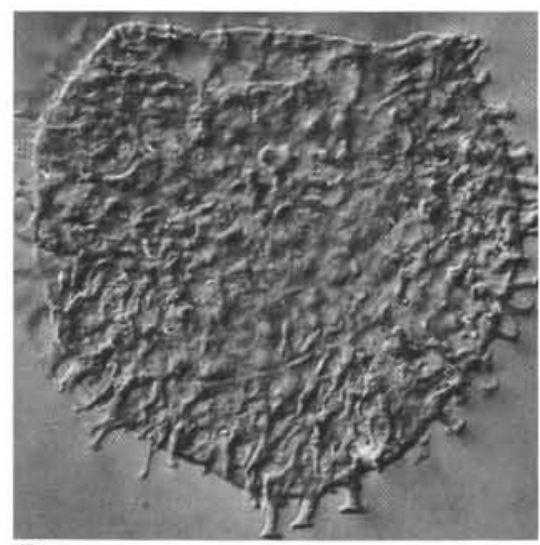

5

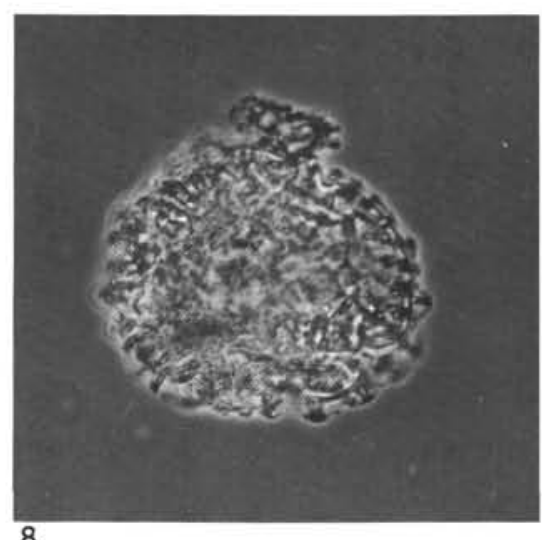

8

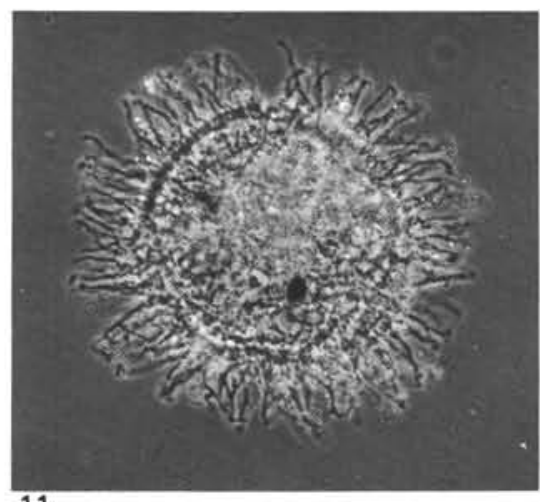

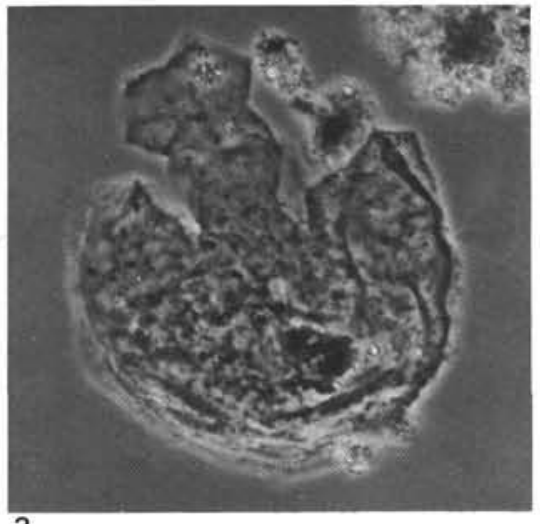

3

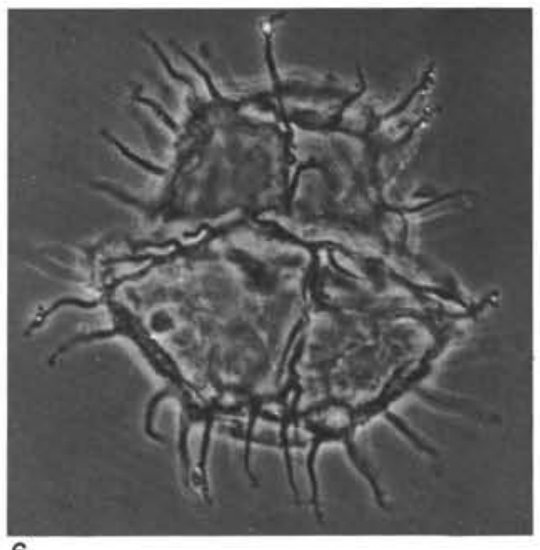

6
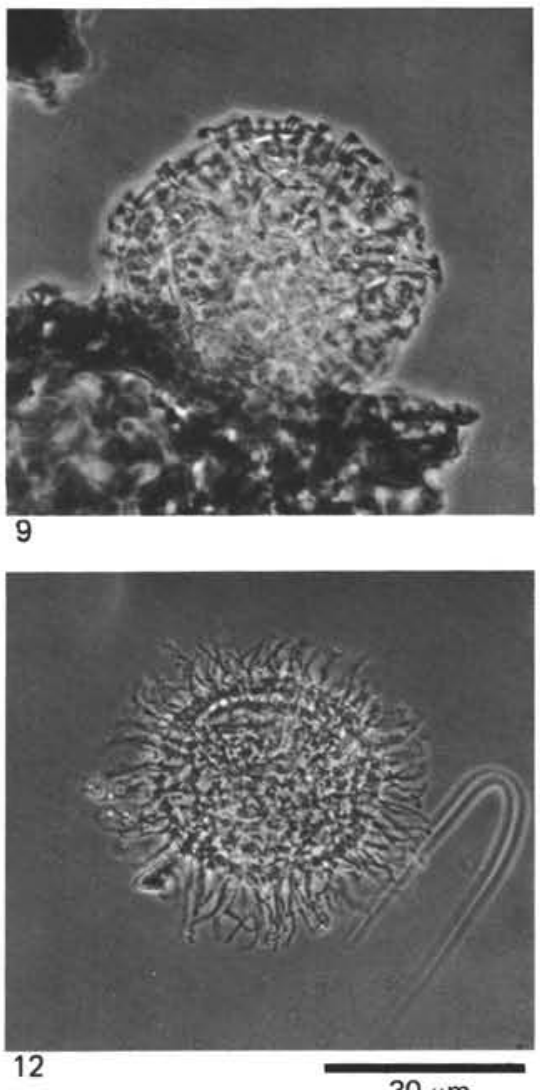


\section{PLATE 4}

Bar on all figures equals $30 \mu \mathrm{m}$.

Figures 1, 2 Oligosphaeridium sp. A. Sample 9-4, 31-35 cm. GSC No. 56728 .

Figure 3 Kleithriasphaeridium eoinodes. Sample 31-3, 70$74 \mathrm{~cm}$. GSC No. 56729.

Figure $4 \quad$ Systematophora sp. cf. S. areolata. Sample 31-3, 70-74 cm. GSC No. 56730 .

Figures 5, $6 \quad$ Systematophora schindewolfii. Sample 16-4, 55$59 \mathrm{~cm}$. GSC No. 56731 .

Figure 7 Systematophora fasciculigera. Sample 51-1, 17-21 cm. GSC No. 56732.

Figure 8 Systematophora sp. A (Habib, 1972). Sample 28-5, 27-30 cm. GSC No. 56733 .

Figure $9 \quad$ Kleithriasphaeridium eoinodes. Sample 30-1, 99$103 \mathrm{~cm}$. GSC No. 56734.

Figure 10 Perisseiasphaeridium sp. A. Sample 14-5, 41-42 cm. GSC No. 56735.

Figure 11 Systematophora sp. Sample 9-4, 31-35 cm. GSC No. 56736 .

Figure 12 Perisseiasphaeridium sp. B. Sample 25-1, 60-66 cm. GSC No. 56737. 
PLATE 4
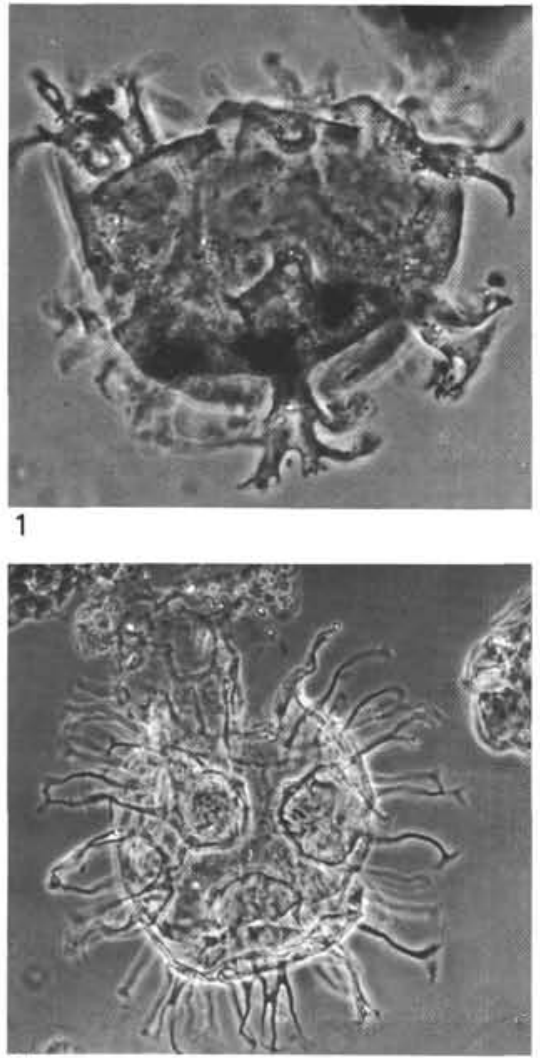

4
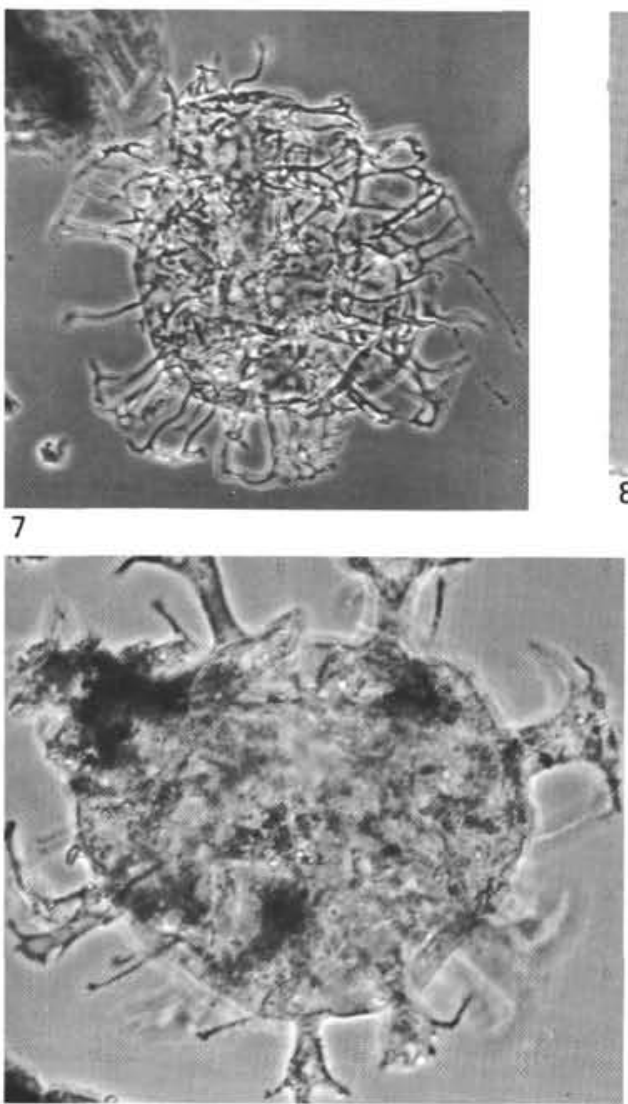

10
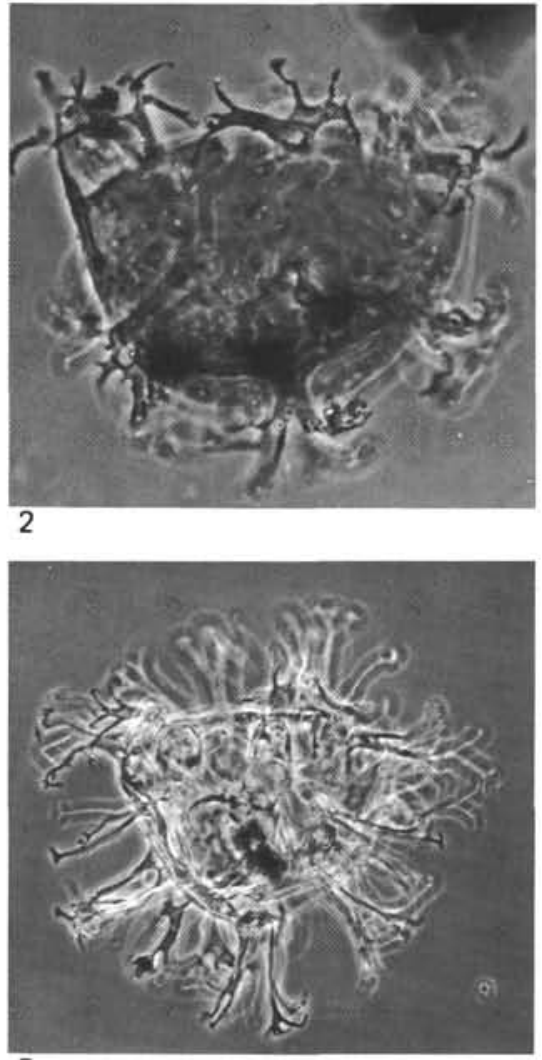

5
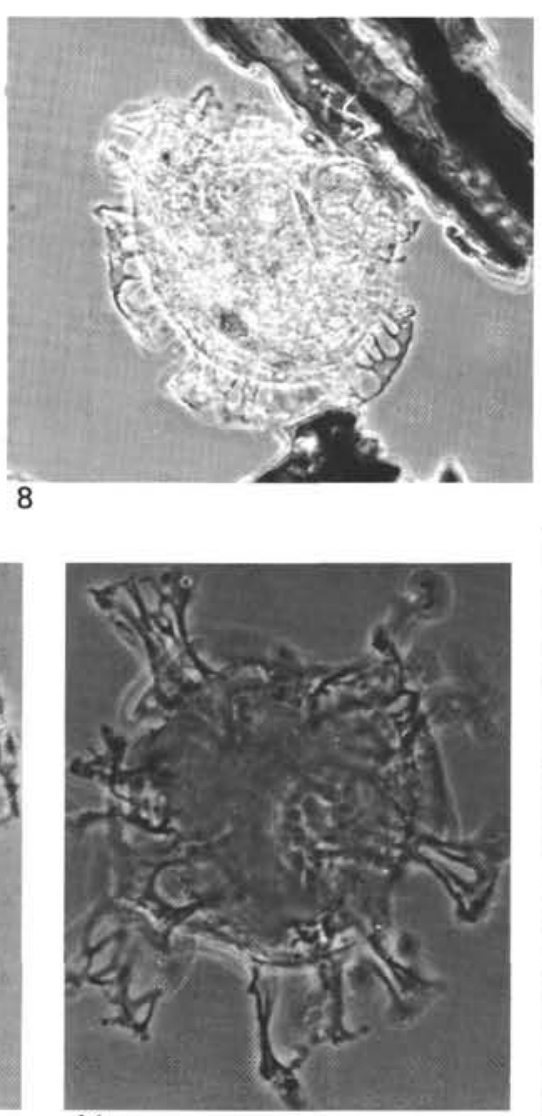

11

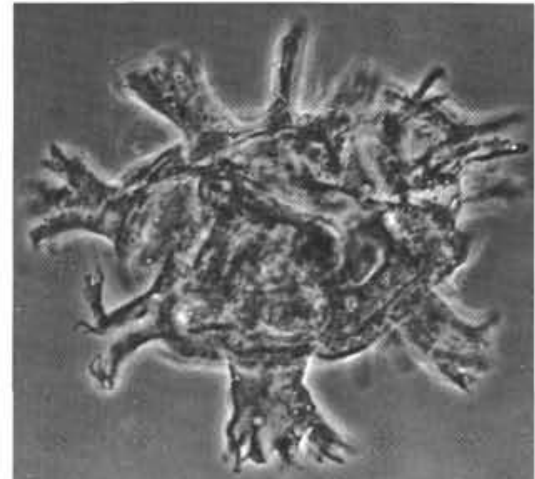

3

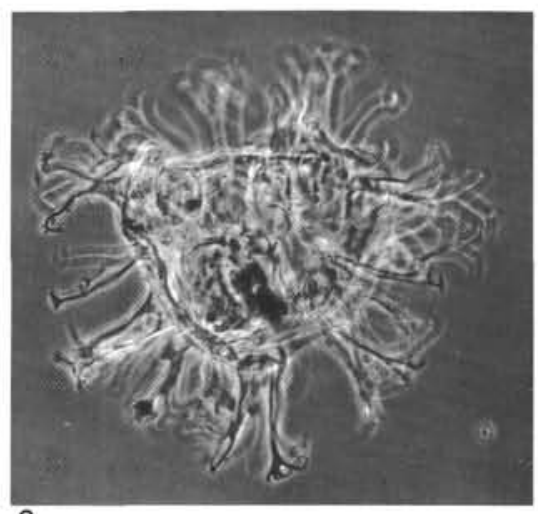

6
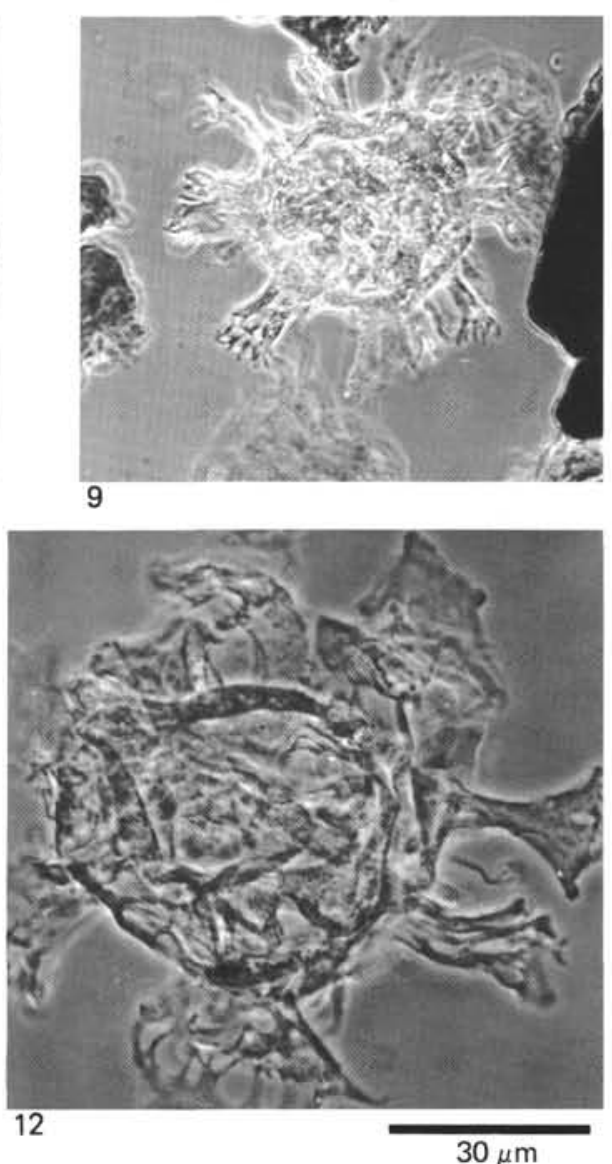


\section{PLATE 5}

Bar on all figures equals $30 \mu \mathrm{m}$.

Figure $1 \quad$ Phoberocysta sp. cf. P. neocomica. Sample 23-1, 107-111 cm. GSC No. 56738 .

Figure 2 Muderongia simplex. Sample 28-1, 30-34 cm. GSC No. 56739.

Figure 3 Muderongia sp. cf. M. simplex. Sample 36-1, $124-128 \mathrm{~cm}$. GSC No. 56740 .

Figure 4 Muderongia simplex. Sample 36-1, 124-128 cm. GSC No. 56741.

Figure $5 \quad$ Muderongia sp. cf. M. simplex. Sample 12-2, 62-66 cm. GSC No. 56742.

Figure 6 Dingodinium cerviculum. Sample 41-1, 96-100 cm. GSC No. 56743.

Figure 7 Achomosphaera neptuni. Sample 31-3, 70-74 cm. GSC No. 56744.

Figure 8 Achomosphaera neptuni. Sample 41-1, 96-100 cm. GSC No. 56745.

Figure 9 Hystrichodinium sp. A (Ioannides et al., 1977). Sample 24-3, 57-61 cm. GSC No. 56746.

Figure 10 Hystrichodinium sp. A (Ioannides et al., 1977). Sample 51-1, 17-21 cm. GSC No. 56747.

Figure 11 Kalyptea monoceras. Sample 25-3, 90-94 cm. GSC No. 56748. 
PLATE 5
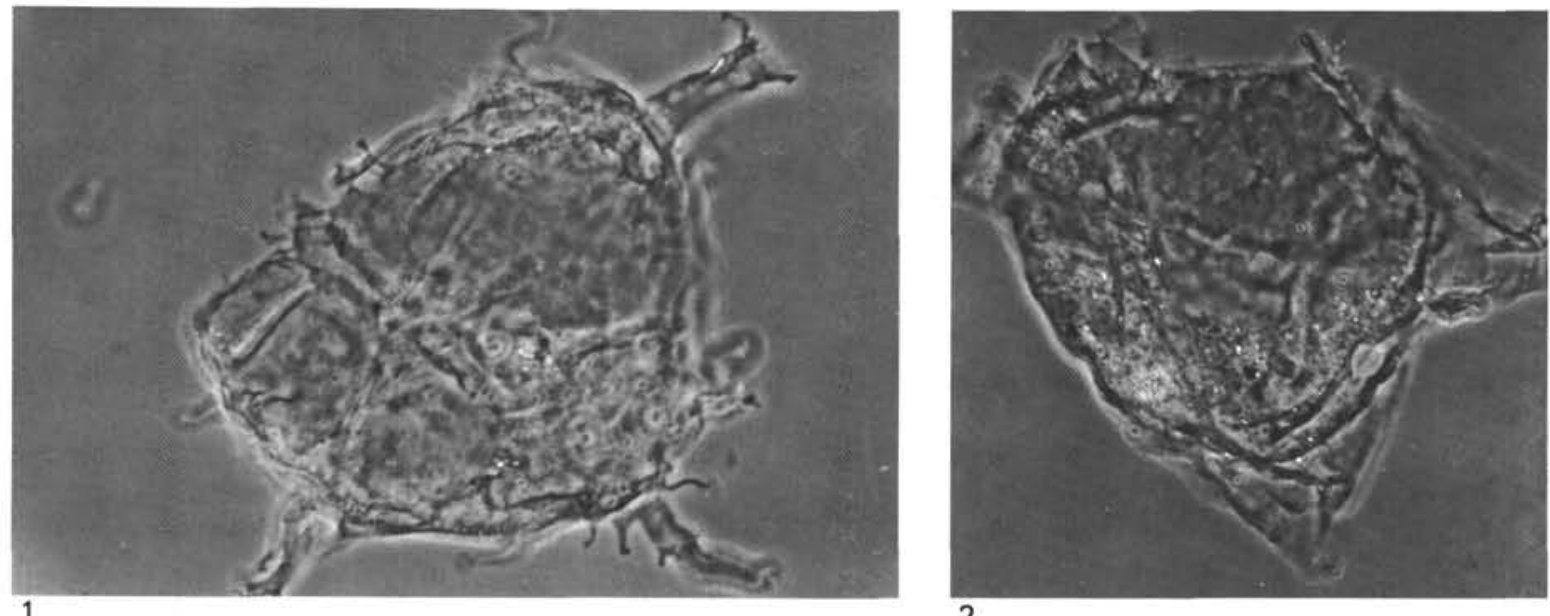

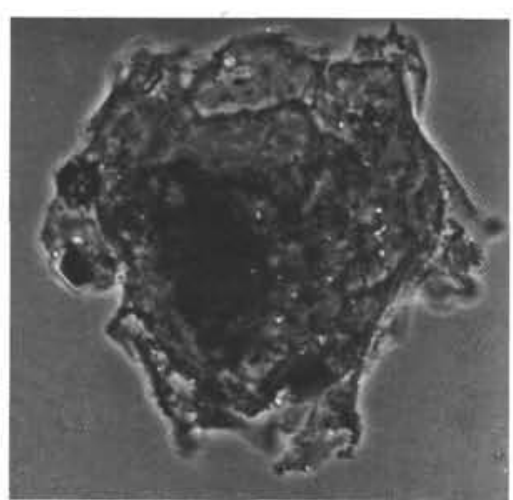

3

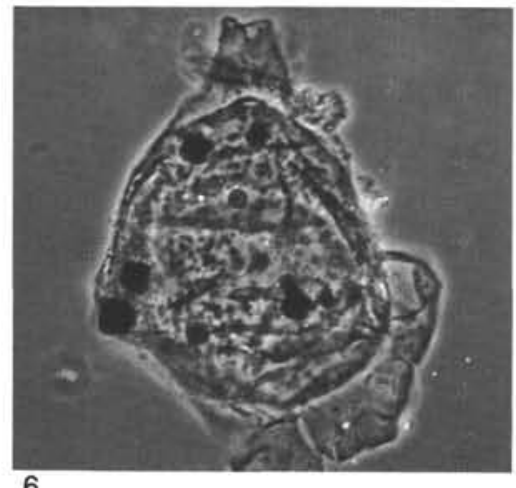

6

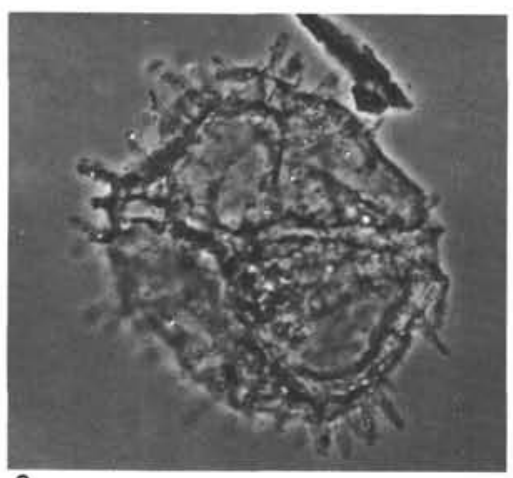

9

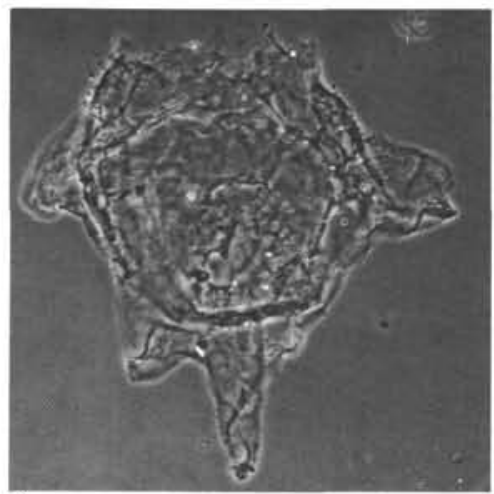

4

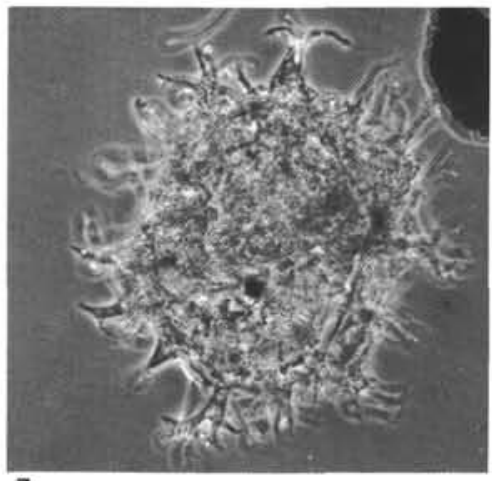

7

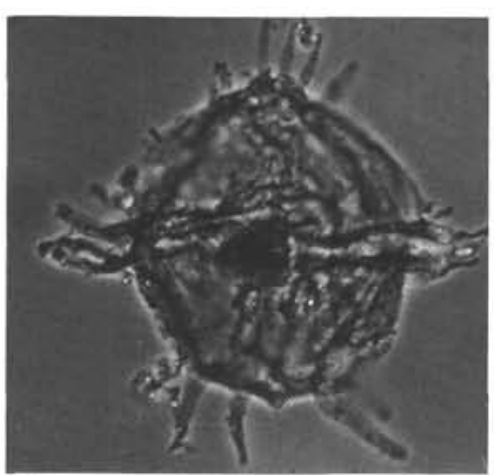

10

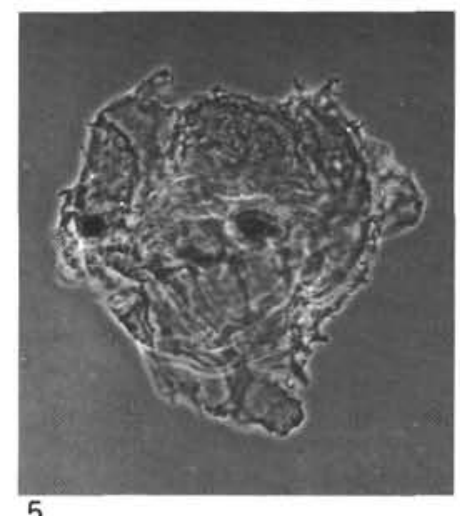

5

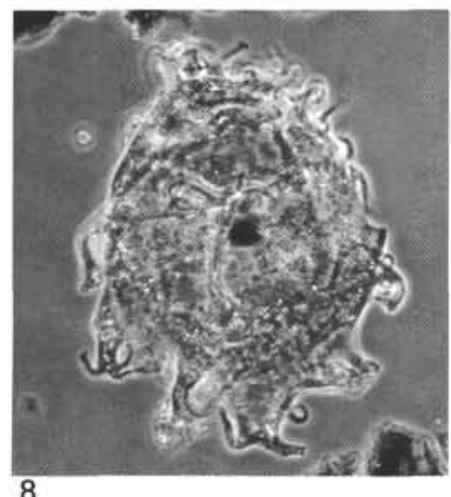

8

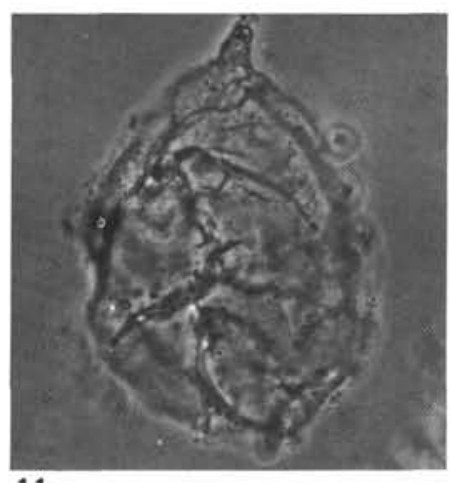

$30 \mu \mathrm{m}$ 


\section{PLATE 6}

Bar on all figures equals $30 \mu \mathrm{m}$.

Figures 1, 2 Tenua sp. A. Sample 12-2, 62-66 cm. GSC No. 56749.

Figures 3, 4 Cyclonephelium distinctum subsp. brevispinatum. Sample 16-4, 55-59 cm. GSC No. 56750 .

Figure 5 Canningia sp. A. Sample 24-3, 57-61 cm. GSC No. 56751.

Figure 6 Canningia sp. A. Sample 24-3, 57-61 cm. GSC No. 56752 . 
PLATE 6

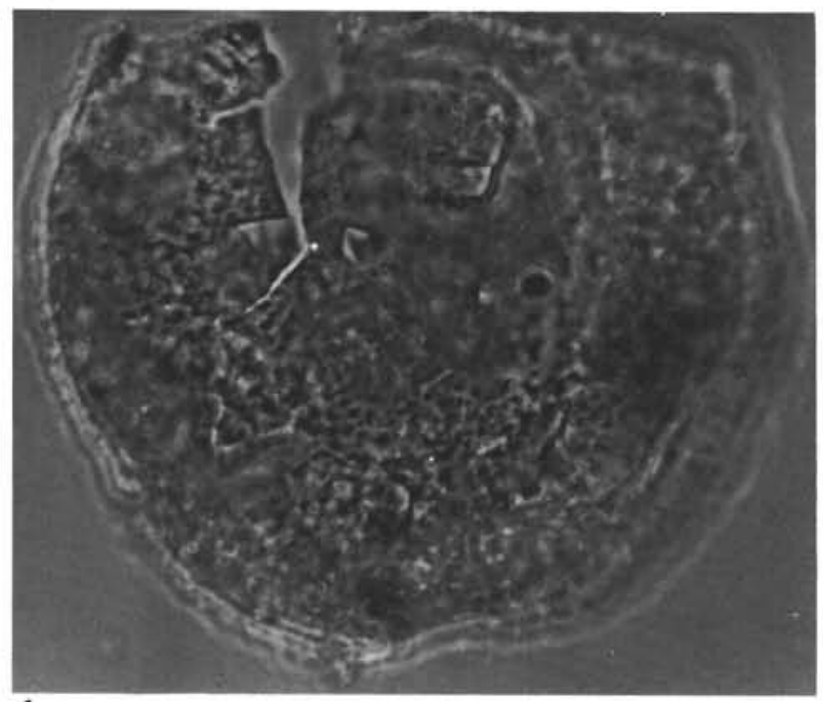

1

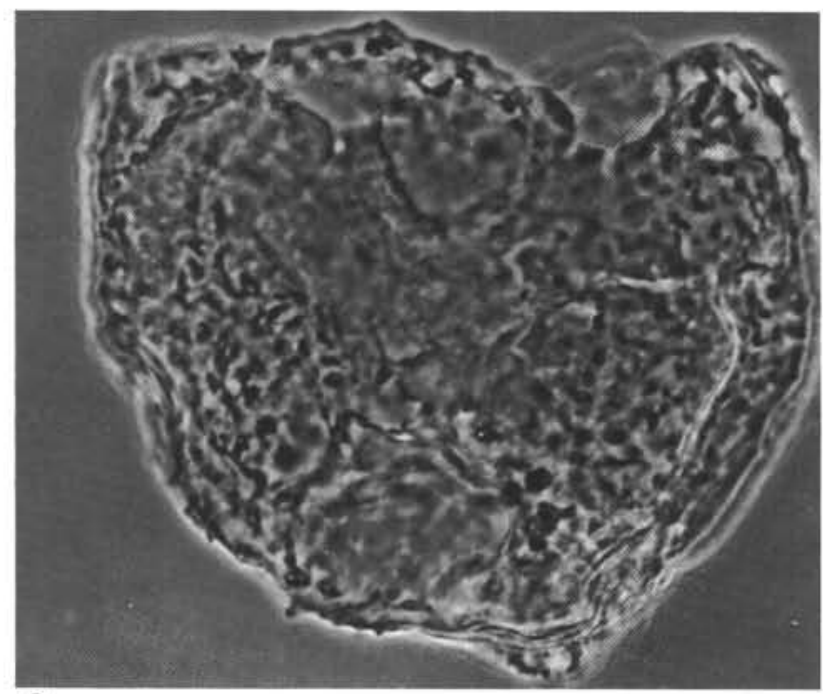

3

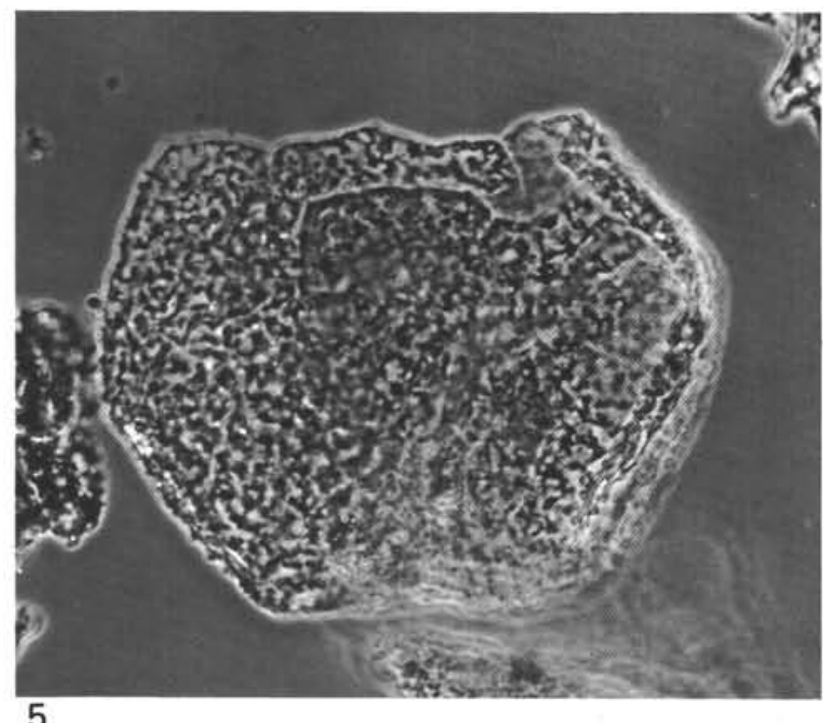

5

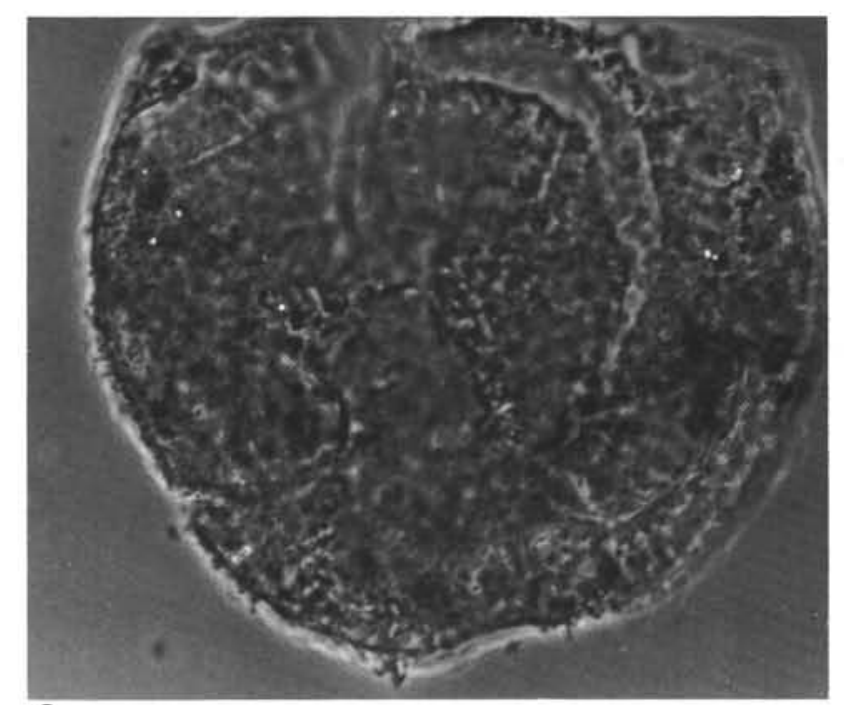

2
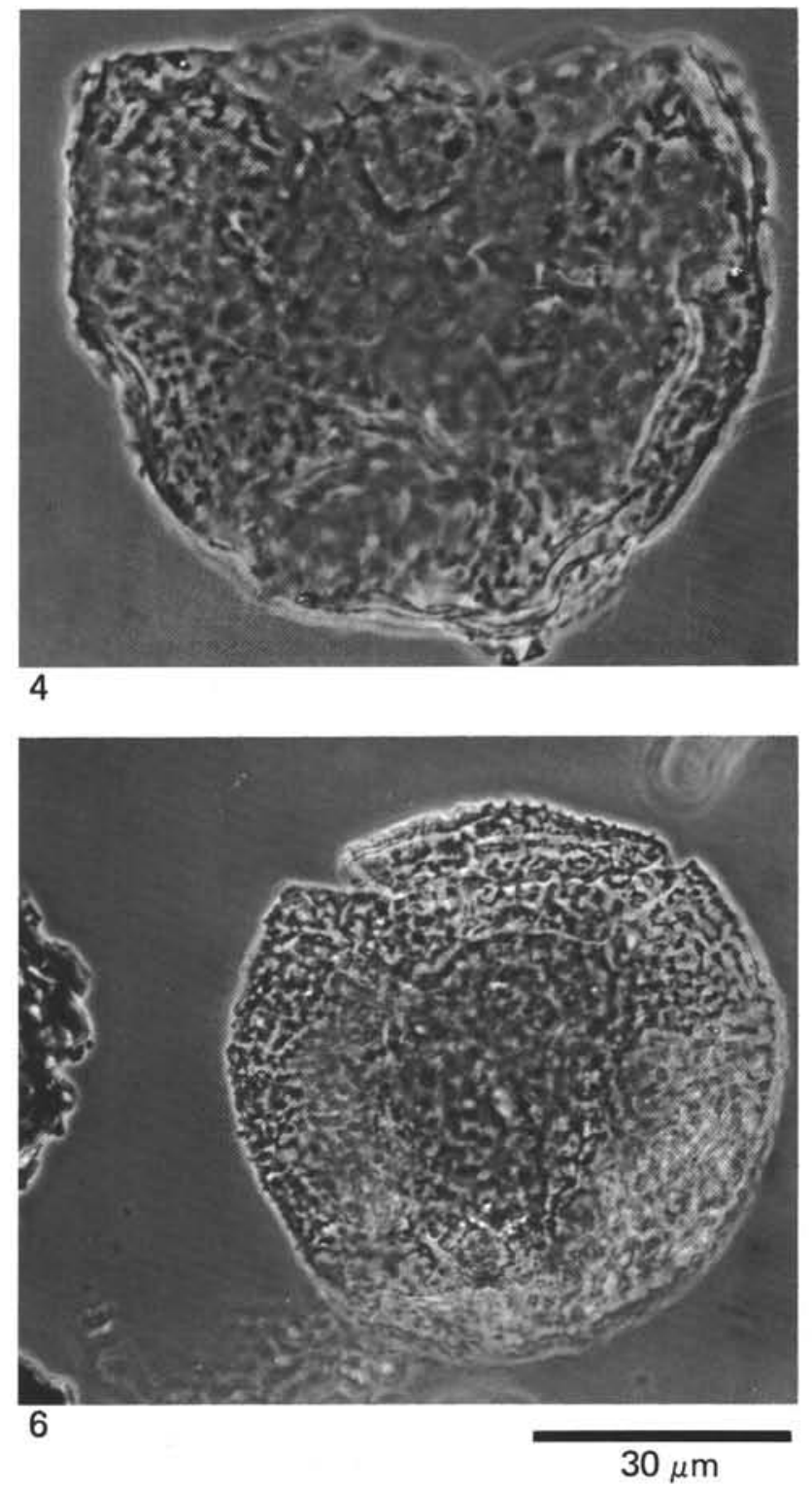


\section{PLATE 7}

Bar on all figures equals $30 \mu \mathrm{m}$.

Figure 1 Cyclonephelium distinctum. Sample 31-3, 70-74 cm. GSC No. 56753.

Figure 2 Tenua hystrix. Sample 31-3, 70-74 cm. GSC No. 56754.

Figure 3 Specimen intermediate between Cyclonephelium distinctum and Tenua hystrix. Sample 31-3, 70-74 cm. GSC No. 56755.

Figure 4 Cyclonephelium distinctum. Sample 31-3, 70-74 cm. GSC No. 56756.

Figure 5 Specimen of Tenua hystrix showing partial delineation of paraplates by spines. Sample 31-3, 70$74 \mathrm{~cm}$. GSC No. 56757.

Figure 6 Systematophora sp. cf. S. areolata. Sample 31-3, 70-74 cm. GSC No. 56758. 
PLATE 7

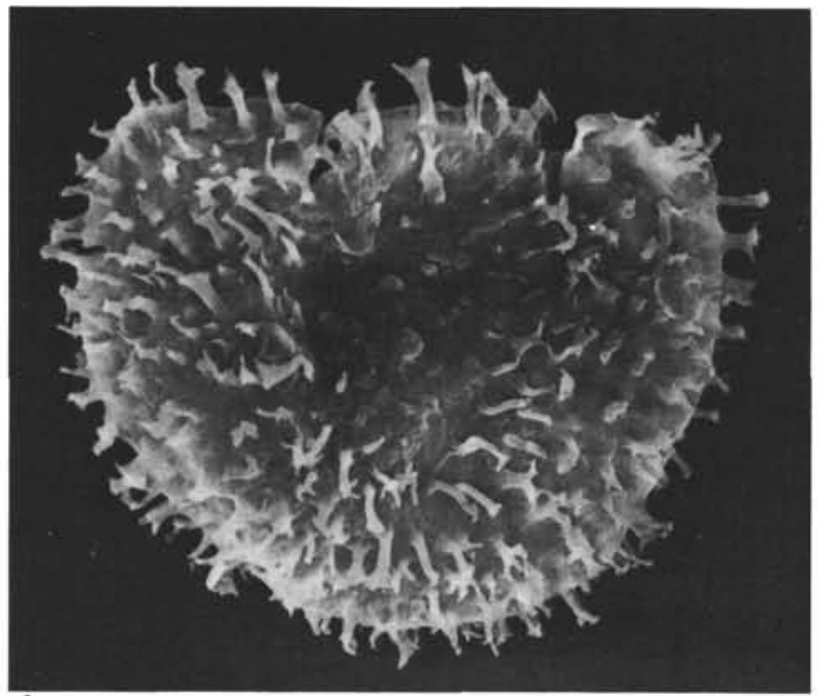

1

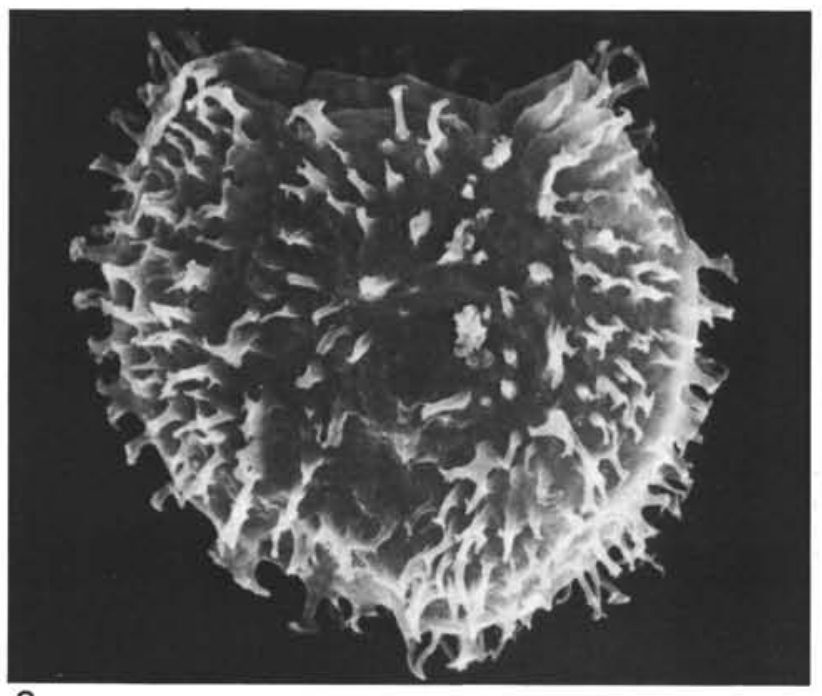

3

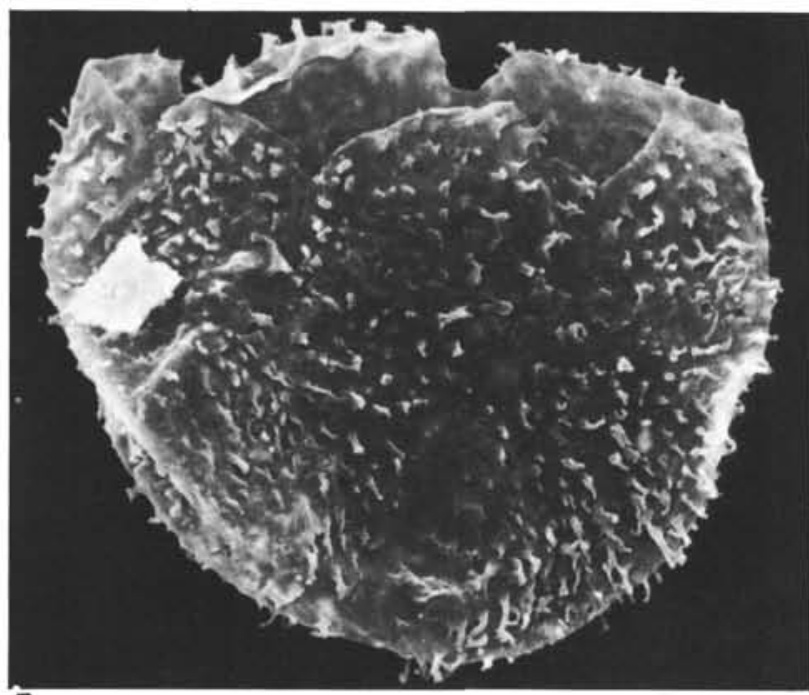

5

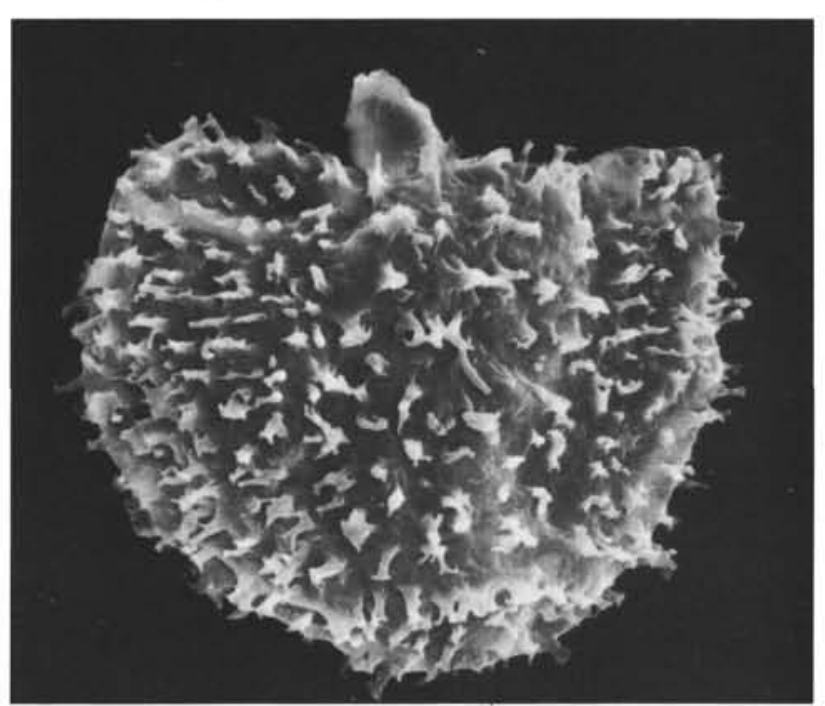

2

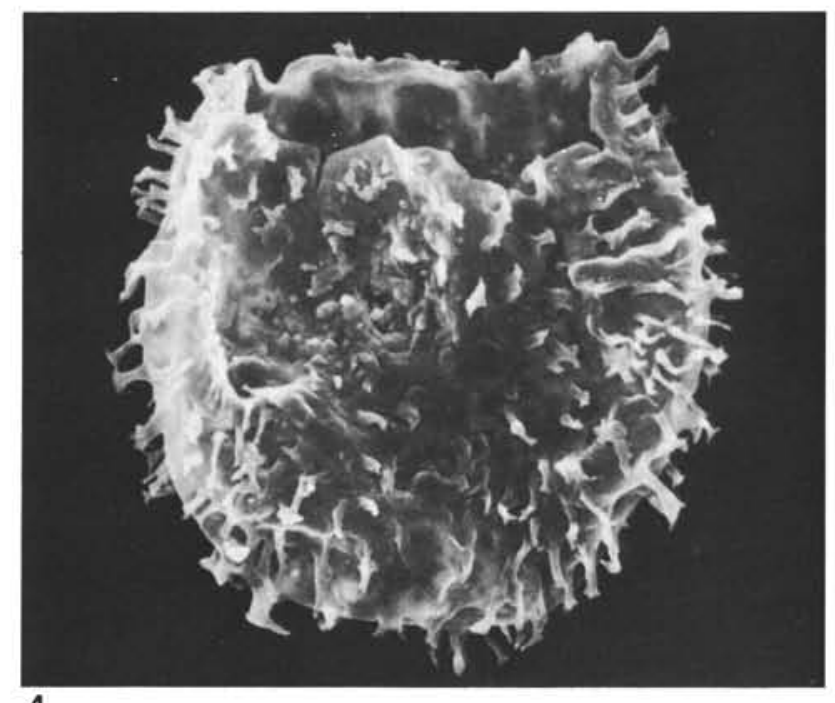

4

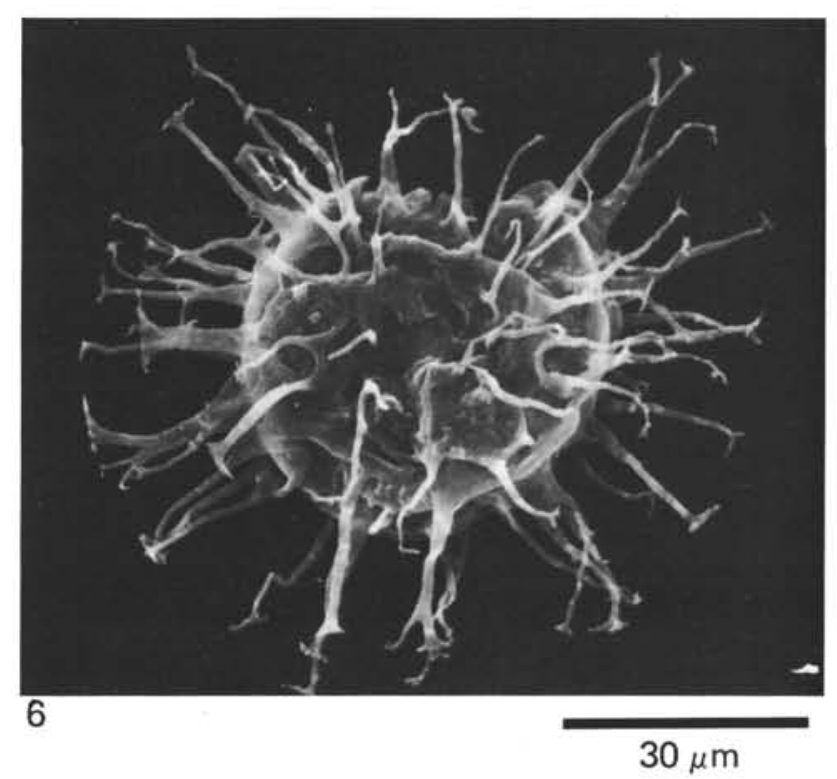




\section{PLATE 8}

Bar on all figures equals $30 \mu \mathrm{m}$.

Figure 1 Polysphaeridium multispinosum. Sample 31-3, 70-74 cm. GSC No. 56759.

Figure 2 Polysphaeridium sp. A. Sample 31-3, 70-74 cm. GSC No. 56760.

Figure 3 Tanyosphaeridium boletum. Sample 31-3, 70-74 cm. GSC No. 56761.

Figure $4 \quad$ Polysphaeridium sp. A. Sample 31-3, 70-74 cm. GSC No. 56762.

Figure $5 \quad$ Polysphaeridium sp. A. Sample 31-3, 70-74 cm. GSC No. 56763.

Figure 6 Prolixosphaeridium granulosum. Sample 31-3, 70-74 cm. GSC No. 56764 . 
PLATE 8
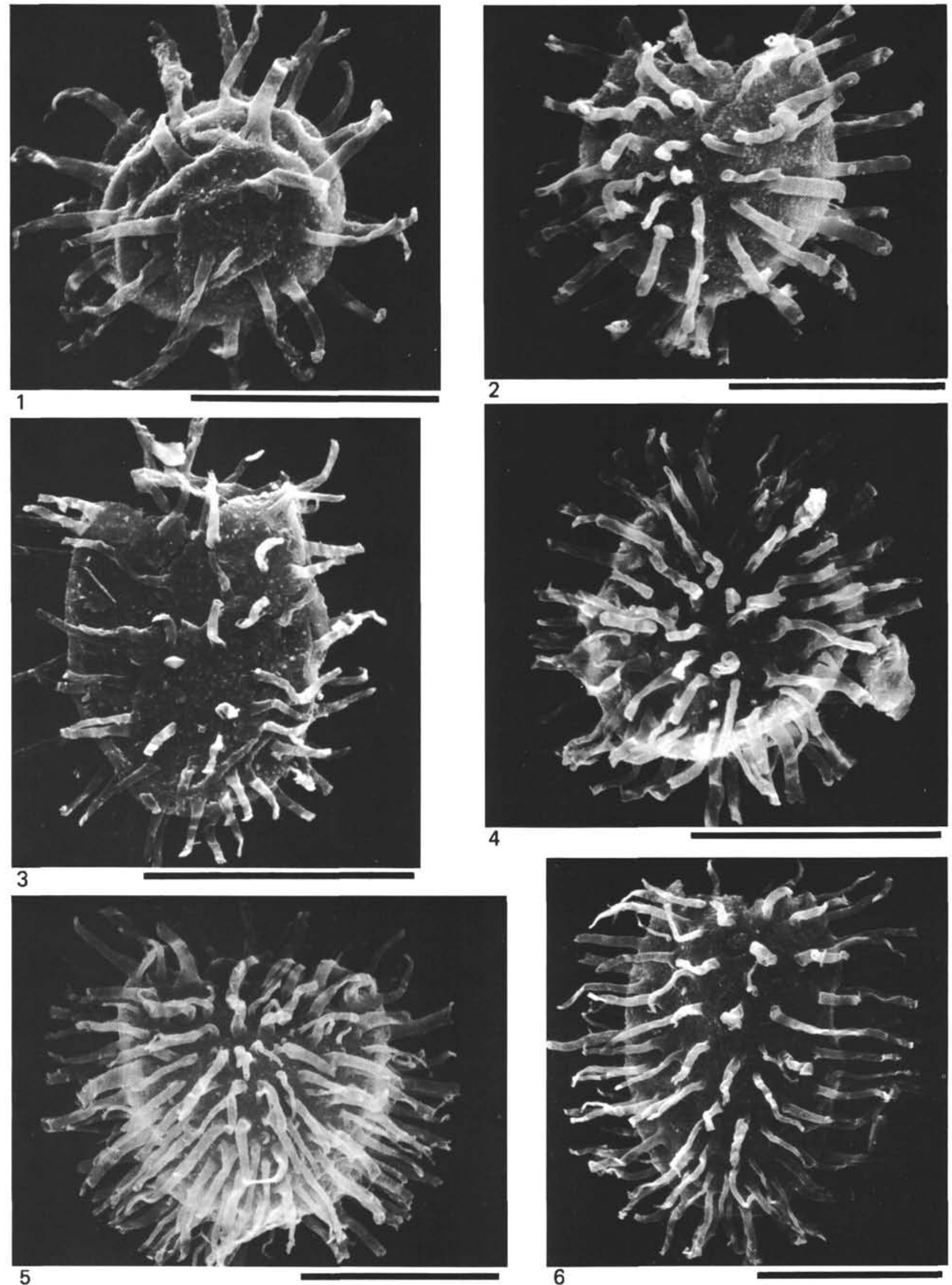


\section{PLATE 9}

Bar on all figures equals $30 \mu \mathrm{m}$.

Figure 1 Gonyaulacysta fastigiata. Sample 31-3, 70-74 cm. GSC No. 56765 .

Figure 2 Gonyaulacysta fastigiata. Polar view of autapex. Sample 31-3, 70-74 cm. GSC No. 56766.

Figure 3 Achomosphaera neptuni. Sample 31-3, 70-74 cm. GSC No. 56767.

Figure 4 Cometodinium sp. A (Habib, 1972). Sample 31-3, $70-74 \mathrm{~cm}$. GSC No. 56768 .

Figure 5 Pseudoceratium pelliferum with a strongly reduced postcingular horn. Sample 31-3, 70-74 cm. GSC No. 56769.

Figure 6 Cometodinium sp. A (Habib, 1972). Sample 31-3, 70-74 cm. GSC No. 56770 . 
PLATE 9
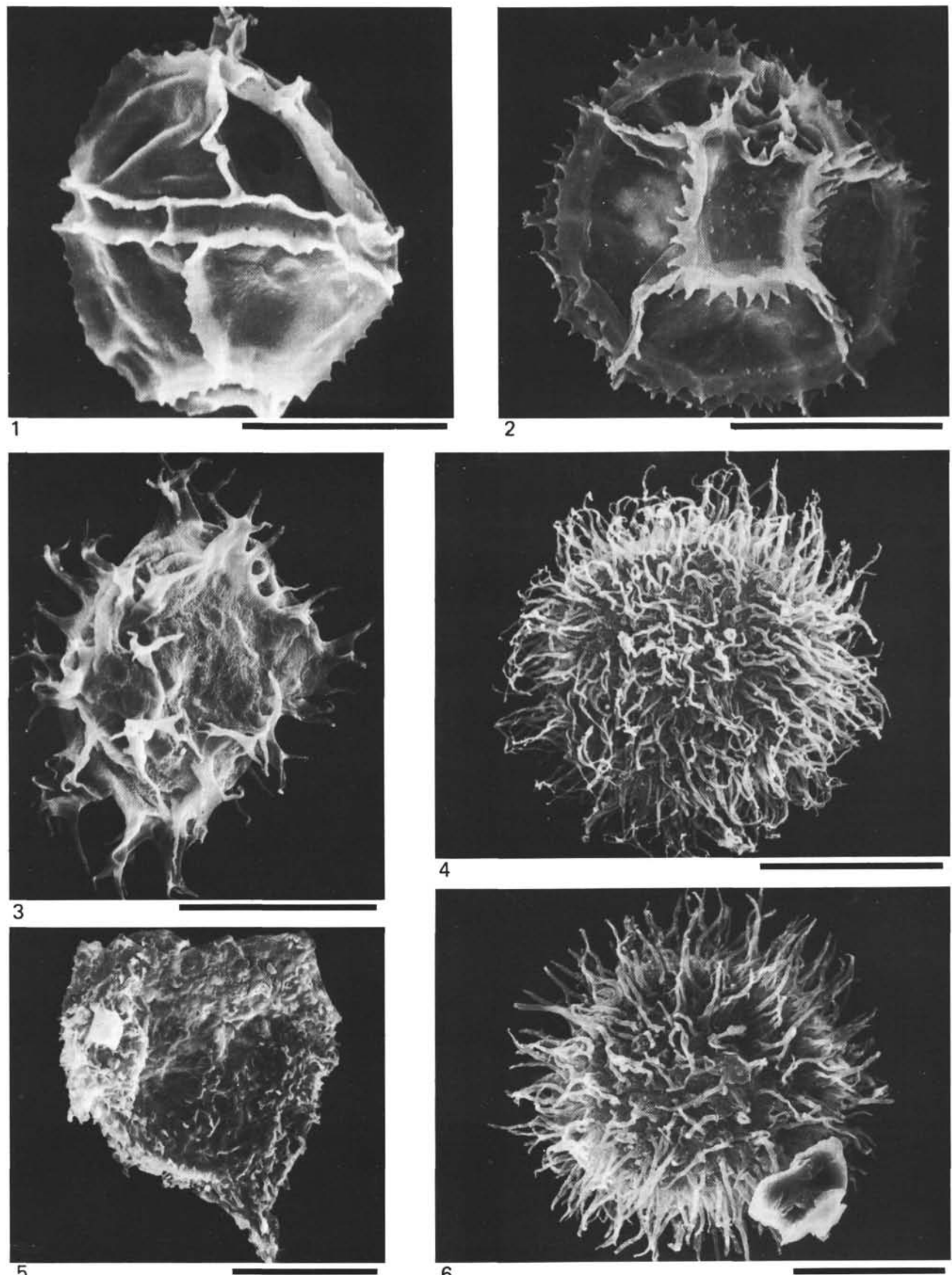
PLATE 10

Bar on all figures equals $30 \mu \mathrm{m}$.

Figures 1, 2 Aequitriradites verrucosus. Sample 24-3, 57-61 cm. GSC No. 56771.

Figure 3 Aequitriradites verrucosus. Sample 41-4, 70-75 cm. GSC No. 56772.

Figure 4 Cicatricosisporites hughesi. Sample 24-3, 57-61 cm. GSC No. 56773.

Figure $5 \quad$ Cicatricosisporites hughesi. Sample 16-4, 55-59 cm. GSC No. 56774.

Figure 6 Cicatricosisporites sp. Sample 11-5, 19-23 cm. GSC No. 56775.

Figure 7 Ephedripites sp. A. Sample 24-3, 57-61 cm. GSC No. 56776 .

Figure $8 \quad$ Cicatricosoporites auritus. Sample 16-4, 55-59 cm. GSC No. 56777.

Figure 9 Cicatricososporites auritus. Sample 24-3, 57-61 cm. GSC No. 56778.

Figure 10 Contignisporites cooksonii. Sample 51-1, 17-21 cm. GSC No. 56779.

Figure 11 Appendicisporites concentricus. Sample 17-1, 65-69 cm. GSC No. 56780.

Figure 12 Verrucosisporites sp. Sample 11-1, 64-68 cm. GSC No. 56781. 
PALYNOLOGICAL STRATIGRAPHY

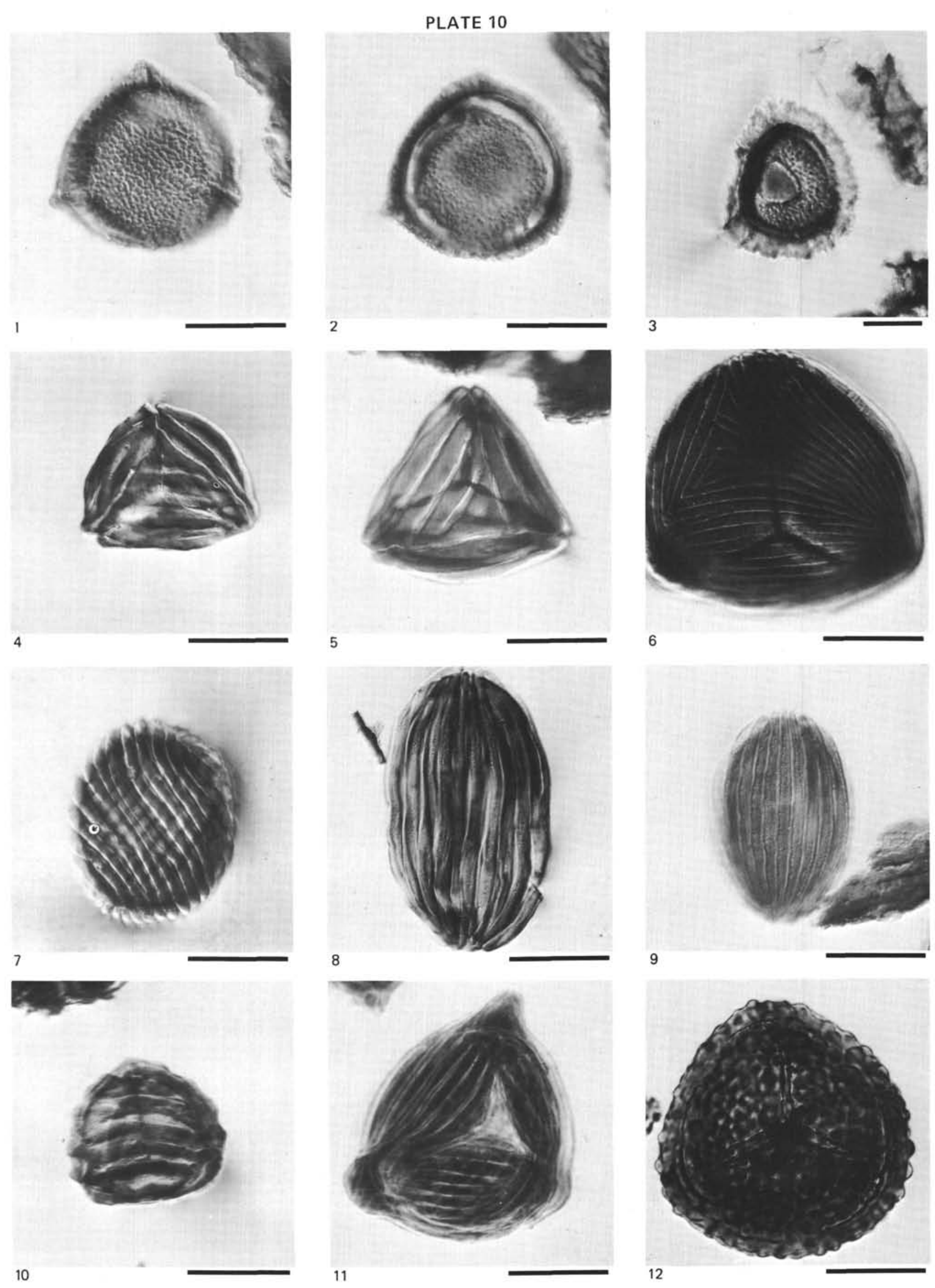


PLATE 11

Bar on all figures equals $50 \mu \mathrm{m}$.

Figure 1 Pilosisporites sp. A. Sample 13-1, 138-141 cm. GSC No. 56782.

Figure 2 Pilosisporites sp. A. Sample 12-4, 112-116 cm. GSC No. 56783.

Figure 3 Pilosisporites sp. A. Sample 19-5, 82-86 cm. GSC No. 56784.

Figure $4 \quad$ Triletes sp. A. Sample 15-3, 54-58 cm. GSC No. 56785.

Figure 5 Trilobosporites jurassicus. Sample 17-1, 65-69 cm. GSC No. 56786.

Figure 6 Callialasporites trilobatus. Sample 16-4, 55-59 cm. GSC No. 56787.

Figure 7 Leptolepidites verrucatus. Sample 24-3, 57-61 cm. GSC No. 56788.

Figure 8 Verrucosisporites sp. Sample 11-5, 19-23 cm. GSC No. 56789.

Figure 9 Leptolepidites psarosus. Sample 34-1, 63-67 cm. GSC No. 56790.

Figure 10 Rouseisporites reticulatus. Sample 22-3, 38-41 cm. GSC No. 56791.

Figure 11 Klukisporites sp. A. Sample 41-4, 70-75 cm. GSC No. 56792.

Figure 12 Klukisporites sp. A. Sample 51-1, 17-21 cm. GSC No. 56793. 


\section{PLATE 11}
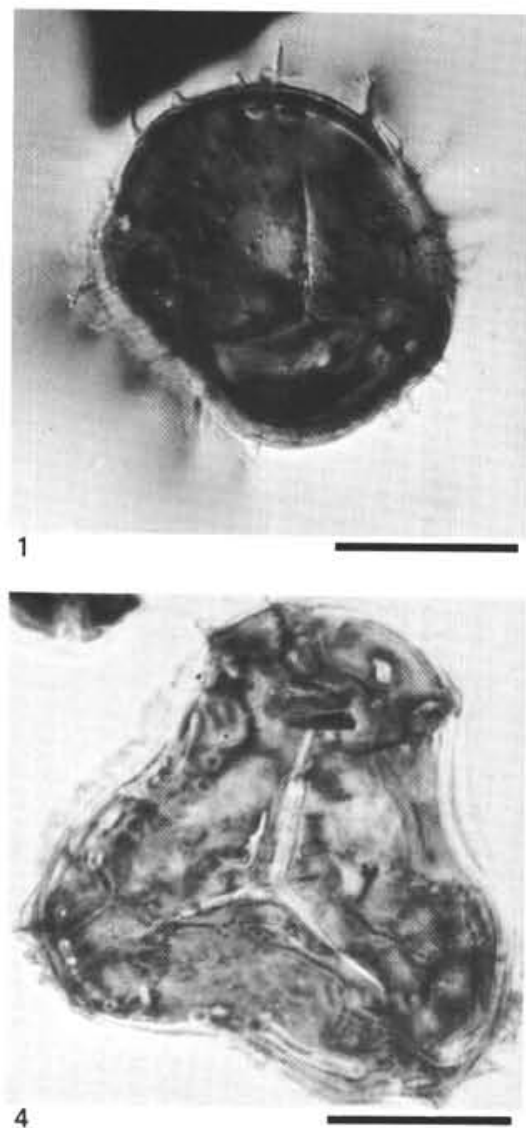

4
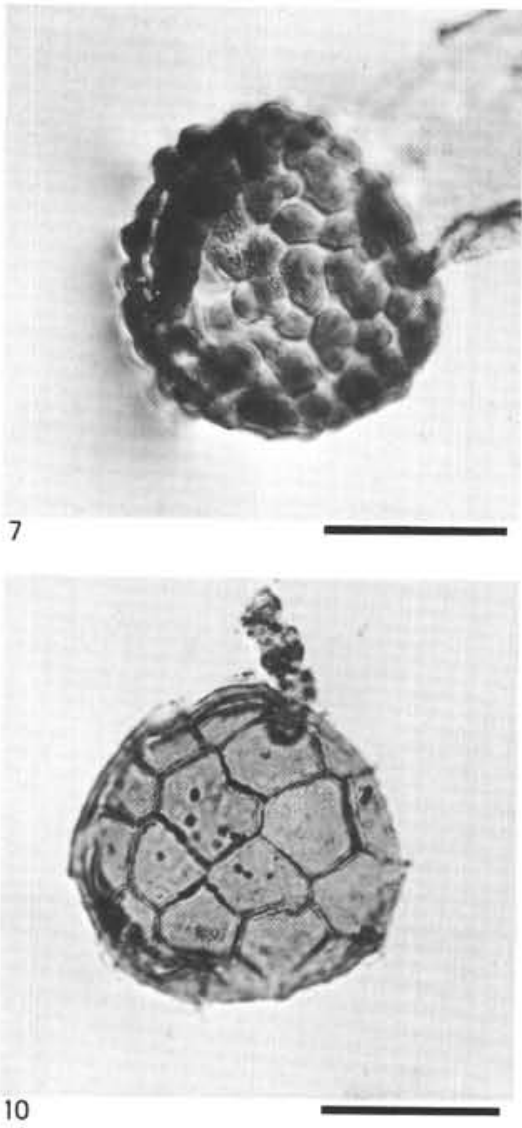
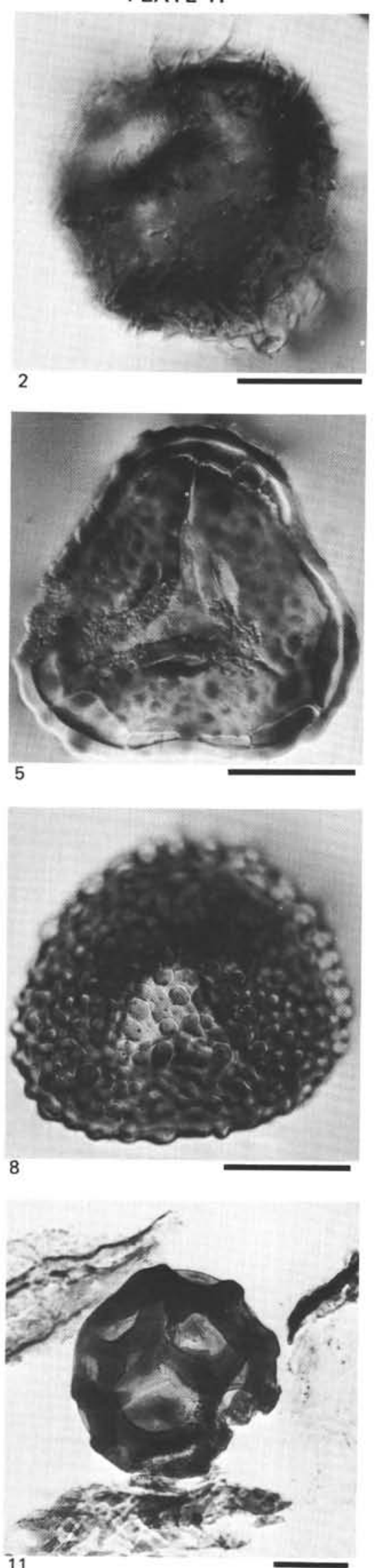
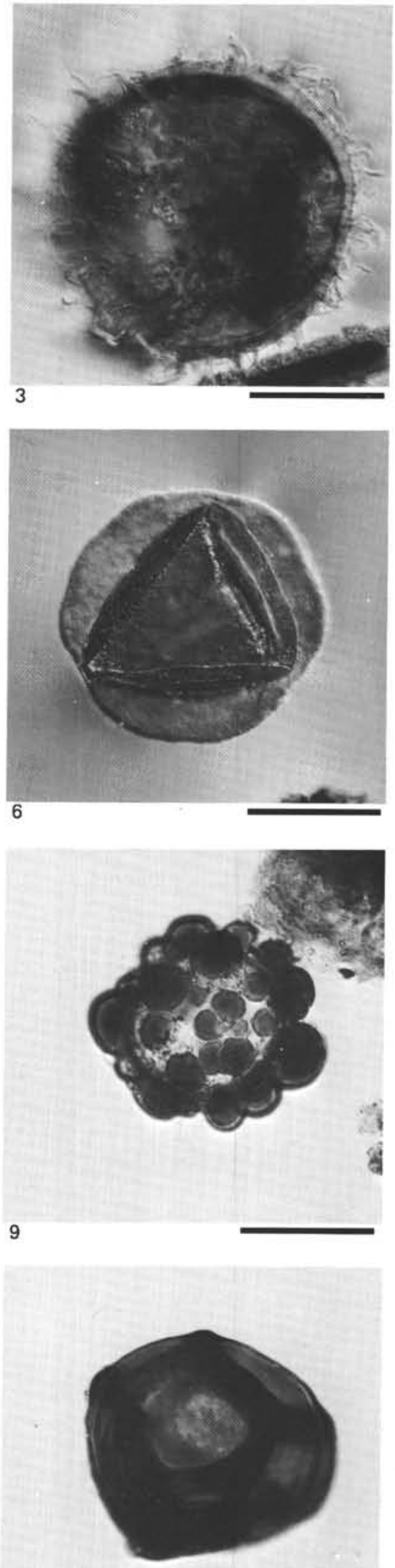